\title{
Random social choice functions for single-peaked domains on trees
}

Citation for published version (APA):

Peters, H., Roy, S., \& Sadhukhan, S. (2018). Random social choice functions for single-peaked domains on trees. Maastricht University, Graduate School of Business and Economics. GSBE Research Memoranda No. 004 https://doi.org/10.26481/umagsb.2018004

Document status and date:

Published: 08/02/2018

DOI:

10.26481/umagsb.2018004

Document Version:

Publisher's PDF, also known as Version of record

\section{Please check the document version of this publication:}

- A submitted manuscript is the version of the article upon submission and before peer-review. There can be important differences between the submitted version and the official published version of record.

People interested in the research are advised to contact the author for the final version of the publication, or visit the DOI to the publisher's website.

- The final author version and the galley proof are versions of the publication after peer review.

- The final published version features the final layout of the paper including the volume, issue and page numbers.

Link to publication

\footnotetext{
General rights rights.

- You may freely distribute the URL identifying the publication in the public portal. please follow below link for the End User Agreement:

www.umlib.nl/taverne-license

Take down policy

If you believe that this document breaches copyright please contact us at:

repository@maastrichtuniversity.nl

providing details and we will investigate your claim.
}

Copyright and moral rights for the publications made accessible in the public portal are retained by the authors and/or other copyright owners and it is a condition of accessing publications that users recognise and abide by the legal requirements associated with these

- Users may download and print one copy of any publication from the public portal for the purpose of private study or research.

- You may not further distribute the material or use it for any profit-making activity or commercial gain

If the publication is distributed under the terms of Article $25 \mathrm{fa}$ of the Dutch Copyright Act, indicated by the "Taverne" license above, 
Hans Peters, Souvik Roy, Soumyarup Sadhukhan

Random Social Choice Functions for Single-Peaked Domains on Trees

$\mathrm{RM} / 18 / 004$

\section{GSBE}

Maastricht University School of Business and Economics

Graduate School of Business and Economics

P.O Box 616

NL-6200 MD Maastricht

The Netherlands 


\title{
Random Social Choice Functions for Single-PEAKED DOMAINS ON TREeS*
}

\author{
Hans Peters ${ }^{\dagger} \quad$ Souvik Roy ${ }^{\ddagger} \quad$ Soumyarup Sadhukhan ${ }^{\S}$ \\ February 1, 2018
}

\begin{abstract}
Finitely many agents have single-peaked preferences on a finite set of alternatives structured as a tree. Under a richness condition on the domain we characterize all unanimous and strategy-proof random social choice functions. These functions are uniquely determined by the values they assign to preference profiles where all peaks are on leafs of the tree.
\end{abstract}

JEL Classification: D71.

Keywords: random social choice function, single-peaked domain, trees, strategy-proofness

\section{Introduction}

Finitely many agents have preferences over a finite set of alternatives. The alternatives are the vertices in a tree, and preferences are linear orderings which are single-peaked: there is a single peak (top alternative) and preference decreases along paths away from this peak. Moreover, it is assumed that the domain of admissible single-peaked preferences is sufficiently rich; this includes the condition of top-connectedness, meaning that for every pair of alternatives $a$ and $b$ between which there is an edge, there is a preference with $a$ at top and $b$ second.

We consider random social choice functions: these assign a probability distribution over the alternatives to every profile of preferences. The conditions we impose are unanimity and strategy-proofness. Unanimity means that if the agents share a common peak then that alternative gets probability one. Strategy-proofness means that no agent, by misrepresenting its true preference, can increase the probability on any upper contour set, i.e., any set of alternatives (weakly) preferred to some given alternative. Put differently, the probability distribution attained by reporting truthfully stochastically dominates any probability distribution achievable by misreporting.

\footnotetext{
*The authors would like to thank Huaxia Zeng and Arunava Sen for their helpful suggestions.

${ }^{\dagger}$ Department of Quantitative Economics, School of Business and Economics, Maastricht University. Email: h.peters@maastrichtuniversity.nl

${ }^{\ddagger}$ Economic Research Unit, Indian Statistical Institute, Kolkata. Email: souvik.2004@gmail.com

$\S$ Economic Research Unit, Indian Statistical Institute, Kolkata.

Email: soumyarup.sadhukhan@gmail.com
} 
Our main result is that a random social choice function satisfies these two conditions if and only if it is tree-monotonic. In a nutshell, this means that such a random social choice function is uniquely determined by the probability distributions it assigns to preference profiles with all peaks at the leafs (terminal nodes) of the tree. We show that such a collection of probability distributions has the following properties: (i) a leaf gets probability one if all peaks are at this leaf (ii) if an agent changes its peak from one leaf to another, then (a) probability increases along the path from the former to the latter and (b) probability does not change off this path. Conversely, every such collection determines a random social choice function that is unanimous and strategy-proof.

The paper thus generalizes the case where the alternatives are ordered on a straight line and agents have single-peaked preferences: see below for a short discussion of this literature. As an application, consider a road or railroad network in the form of a tree (especially in the railroad case this is not a far-fetched assumption), where the vertices (junctions) are also the locations of villages or cities. The objective is to locate a public good (shopping mall, museum, etc.) based on the preferences of the agents over these junctions - single-peakedness is then a plausible assumption.

The literature on random social choice functions started with the paper of Gibbard (1977). Without restrictions on preferences, the conditions of unanimity and strategy-proofness result in random dictatorships - that is, fixed probability distributions over the top alternatives of the agents. The single-peaked domain restriction allows for other functions, which can be seen as probabilistic extensions of the generalized median functions (Moulin, 1980; Barberà et al., 1993; and others): see Ehlers et al. (2002) for the case with finitely many agents who have single-peaked preferences over the real line. In Peters et al. (2014) it is shown for a discrete version of this model (finitely many alternatives on the real line) that all random social choice functions are convex combinations of deterministic functions. In the concluding Section 5 we show that in the current setting this is no longer the case, by providing an example of a random tree-monotonic social choice function which is not a convex combination of deterministic tree-monotonic social choice functions. The current paper can be seen as generalizing, albeit for a finite set of alternatives, the results in Ehlers et al. (2002) to the situation where the alternatives are structured on a tree. Chatterji et al. (2016) show a kind of converse: a domain has to be single-peaked in order to allow for the existence of unanimous and strategy-proof random social choice functions satisfying two additional conditions. ${ }^{1}$ See also Chatterji and Massó (2015) for a similar result in the deterministic case.

After preliminaries in Section 2, we show in Section 3 that on a rich single-peaked domain any unanimous and strategy-proof random social choice function is uncompromising (cf. Border and Jordan, 1983): changing one's peak can only change the probabilities on the path from the old towards the new peak, but not those off this path. While this is a relatively easy result in the real-line case, its proof is rather cumbersome in the tree-case. Using this result (Theorem 3.1) we show in Section 4 that the unanimous and strategy-proof random social choice functions are exactly the tree-monotonic social choice functions briefly described above (Theorem 4.1). Section 5 concludes.

\footnotetext{
${ }^{1}$ Namely, tops-onliness and a 'compromise' property. Under the assumptions in our paper tops-onliness follows from the other conditions. The 'compromise' property is not necessarily satisfied by a tree-monotonic random social choice function.
} 


\section{Preliminaries}

Let $A$ be a finite set of alternatives and let $N=\{1, \ldots, n\}$ be a finite set of agents. A complete, asymmetric, and transitive binary relation over $A$ (also called a linear order) is called a preference. A collection of preferences $P_{N}=\left(P_{1}, \ldots, P_{n}\right)$ is called a preference profile. We denote by $\mathbb{L}(A)$ the set of all preferences over $A$. A domain (of admissible preferences) is a subset of $\mathbb{L}(A)$.

For a preference $P \in \mathbb{L}(A)$ we denote the top alternative by $t(P)$, i.e., $t(P)=a$ if and only if $a P x$ for all $x \in A \backslash\{a\}$. The upper contour set of an alternative $a$ at preference $P$ is the set $U(a, P)=\{x \in A \mid x P a$ or $x=a\}$.

\subsection{Domains and their properties}

A domain of central importance in social choice theory is the single-peaked domain. It was originally introduced in Black (1948) and Inada (1964). Here, we consider a generalization.

First, we introduce a graph structure on the set of alternatives. A collection $E \subseteq\{\{a, b\} \mid$ $a, b \in A, a \neq b\}$ is an undirected graph. The elements of $E$ are called edges. The degree of $a \in A$ is the number of edges to which it belongs, i.e., the number $|\{\{x, y\} \in E \mid a \in\{x, y\}\}|$. For $a, b \in A$ a path $[a, b]$ is a sequence of nodes $\left(a_{1}, \ldots, a_{k}\right)$ such that $a_{1}=a, a_{k}=b$, and $\left(a_{i}, a_{i+1}\right) \in E$ for all $i=1, \ldots, k-1$. In this case, by $(a, b]$ we denote the sequence $\left(a_{2}, \ldots, a_{k}\right)$, and by $(a, b)$ the sequence $\left(a_{2}, \ldots, a_{k-1}\right)$. Whenever it is clear from the context, the notations $[a, b],(a, b]$, and $(a, b)$ will also be used to denote the sets of nodes (instead of the sequences) that appear in the path.

A graph $E$ is a tree if for all $a, b \in A$ there is a unique path $[a, b]$. Throughout this paper, we assume that $E$ is an arbitrary but fixed tree. By $A_{L} \subseteq A$, we denote the set of alternatives with degree 1 (also called leafs), and by $A_{J} \subseteq A$, we denote the set of alternatives with degree more than two (also called junction nodes). Thus, the alternatives in $A$ are partitioned into three sets: the leafs, the junction nodes, and the nodes with degree exactly two.

Definition 2.1. A preference $P$ is single-peaked if for all distinct $x, y \in A$ with $y \neq t(P)$,

$$
x \in[t(P), y] \Longrightarrow x P y \text {. }
$$

A domain is single-peaked if each preference in it is single-peaked. ${ }^{2}$

To facilitate our next definitions, we introduce a few pieces of notation. For a path $\left[x_{1}, x_{\ell}\right]=\left(x_{1}, \ldots, x_{\ell}\right)$, we write $P=\left[x_{1}, x_{l}\right] \cdots$ to denote a preference $P$ with $x_{1} P x_{2} P \cdots P x_{\ell}$ $P x$ for all $x \in A \backslash\left[x_{1}, x_{\ell}\right]$. Notations like $P=\cdots\left[x_{1}, x_{\ell}\right] \cdots$ and combinations of these have similar meanings. also, brackets are sometimes left out if confusion is unlikely.

Our next definition introduces a well-known property of a domain.

Definition 2.2. A single-peaked domain $\mathcal{S}$ is top-connected if for every $\{a, b\} \in E$ there exists a preference $P \in \mathcal{S}$ with $P=a b \cdots$.

Peters et al. (2104) introduce a minimal richness condition for a single-peaked domain on a line. Our next definition is a modification of this condition for a single-peaked domain on a tree.

\footnotetext{
${ }^{2}$ For a single-peaked preference, the top alternative is also called the peak.
} 


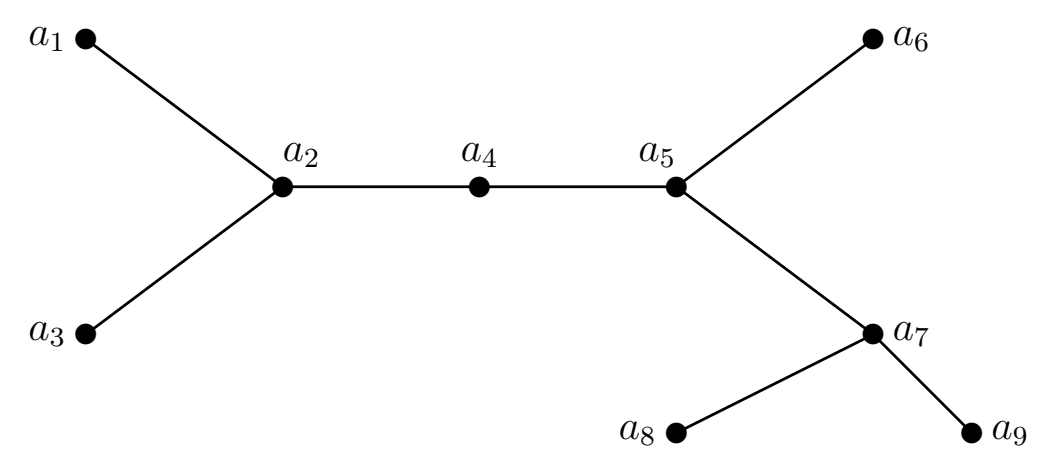

Figure 1: The tree in Example 2.1

Definition 2.3. A single-peaked domain $\mathcal{S}$ is rich if (i) it is top-connected and (ii) for all junction nodes $a \in A_{J}$ and all leafs $b, b^{\prime} \in A_{L}$ such that $a \in\left[b, b^{\prime}\right]$, there exists a preference $P \in \mathcal{S}$ of the form $P=[a, b] \cdots\left(a, b^{\prime}\right] \cdots$ such that for all $c \in A$ for which $a \in[c, b] \cap\left[c, b^{\prime}\right]$, we have $b^{\prime} P c$.

Note that this richness property does not require anything for preferences having a nonjunction node as the top alternative. Thus, a top-connected single-peaked domain on a line is rich. This shows that our richness condition is weaker than the 'minimal richness' condition in Peters et al. (2014). ${ }^{3}$

We illustrate the notion of richness by means of the following example.

Example 2.1. Consider the tree in Figure 1. Consider the non-junction-node $a_{4}$. Then, the richness property requires that there must two preferences $a_{4} a_{5} \cdots$ and $a_{4} a_{2} \cdots$ in the domain with $a_{4}$ at the top. Now, consider the junction-node $a_{5}$. In what follows, we present all the preferences with $a_{5}$ at the top that are required by the richness property. It is easy to see that there are sixteen choices of $b$ and $b^{\prime}$ such that $a_{5} \in\left[b, b^{\prime}\right]$. Therefore, by the richness property, there must be a preference satisfying (ii) in Definition 2.3 for each such choice of $b$ and $b^{\prime}$. In Table 1, we present all these sixteen preference types. The second and the third row in the table provide the choices of $b$ and $b^{\prime}$, respectively. The preferences are presented as columns. For ease of presentation, alternatives outside the paths $\left[a_{5}, b\right]$ and $\left[a_{5}, b^{\prime}\right]$ are not mentioned in the preferences. They are assumed to follow (ii) in Definition 2.3. For instance, in $P_{1}, P_{2}$, and $P_{3}$, the position of $a_{3}$ is unrestricted within the dotted space, and all other alternatives must be below $b^{\prime}$ for those preferences.

\subsection{Social choice functions and their properties}

By $\triangle A$, we denote the set of all probability distributions on $A$. We fix a single-peaked domain $\mathcal{S}$, and denote by $\mathcal{S}^{N}$ the set of all profiles with preferences in $\mathcal{S}$. For $a \in A, \mathcal{S}^{a}$ is the subset of $\mathcal{S}$ of preferences with $a$ at top, i.e., $\mathcal{S}^{a}=\{P \in \mathcal{S} \mid t(P)=a\}$.

A random social choice function (RSCF) is a function $\varphi: \mathcal{S}^{N} \rightarrow \triangle A$. For $B \subseteq A$ and $P_{N} \in \mathcal{S}^{N}$, we write $\varphi_{B}\left(P_{N}\right)=\sum_{a \in B} \varphi_{a}\left(P_{N}\right)$, where $\varphi_{a}\left(P_{N}\right)$ is the probability of $a$ at $\varphi\left(P_{N}\right)$.

\footnotetext{
${ }^{3}$ This condition is as follows. A single-peaked preference (on a line) is called left (right) single-peaked if all the alternatives that are on the left (right) of the peak are preferred to all those that are on the right (left) of the peak. A single-peaked domain is said to be 'minimally rich' if it contains all left single-peaked and all right single-peaked preferences. Clearly, the richness condition in Definition 2.3 does not imply this.
} 


\begin{tabular}{c|cccccccccccccccc} 
& $P_{1}$ & $P_{2}$ & $P_{3}$ & $P_{4}$ & $P_{5}$ & $P_{6}$ & $P_{7}$ & $P_{8}$ & $P_{9}$ & $P_{10}$ & $P_{11}$ & $P_{12}$ & $P_{13}$ & $P_{14}$ & $P_{15}$ & $P_{16}$ \\
\hline$b$ & $a_{1}$ & $a_{1}$ & $a_{1}$ & $a_{3}$ & $a_{3}$ & $a_{3}$ & $a_{8}$ & $a_{8}$ & $a_{8}$ & $a_{9}$ & $a_{9}$ & $a_{9}$ & $a_{6}$ & $a_{6}$ & $a_{6}$ & $a_{6}$ \\
\hline$b^{\prime}$ & $a_{6}$ & $a_{8}$ & $a_{9}$ & $a_{6}$ & $a_{8}$ & $a_{9}$ & $a_{1}$ & $a_{3}$ & $a_{6}$ & $a_{1}$ & $a_{3}$ & $a_{6}$ & $a_{1}$ & $a_{3}$ & $a_{8}$ & $a_{9}$ \\
\hline & $a_{5}$ & $a_{5}$ & $a_{5}$ & $a_{5}$ & $a_{5}$ & $a_{5}$ & $a_{5}$ & $a_{5}$ & $a_{5}$ & $a_{5}$ & $a_{5}$ & $a_{5}$ & $a_{5}$ & $a_{5}$ & $a_{5}$ & $a_{5}$ \\
& $a_{4}$ & $a_{4}$ & $a_{4}$ & $a_{4}$ & $a_{4}$ & $a_{4}$ & $a_{7}$ & $a_{7}$ & $a_{7}$ & $a_{7}$ & $a_{7}$ & $a_{7}$ & $a_{6}$ & $a_{6}$ & $a_{6}$ & $a_{6}$ \\
& $a_{2}$ & $a_{2}$ & $a_{2}$ & $a_{2}$ & $a_{2}$ & $a_{2}$ & $a_{8}$ & $a_{8}$ & $a_{8}$ & $a_{9}$ & $a_{9}$ & $a_{9}$ & $a_{4}$ & $a_{4}$ & $a_{7}$ & $a_{7}$ \\
& $a_{1}$ & $a_{1}$ & $a_{1}$ & $a_{3}$ & $a_{3}$ & $a_{3}$ & $\vdots$ & $\vdots$ & $\vdots$ & $\vdots$ & $\vdots$ & $\vdots$ & $a_{2}$ & $a_{2}$ & $a_{8}$ & $a_{9}$ \\
$\vdots$ & $\vdots$ & $\vdots$ & $\vdots$ & $\vdots$ & $\vdots$ & $a_{4}$ & $a_{4}$ & $a_{6}$ & $a_{4}$ & $a_{4}$ & $a_{6}$ & $a_{1}$ & $a_{3}$ & $\vdots$ & $\vdots$ \\
& $a_{6}$ & $a_{7}$ & $a_{7}$ & $a_{6}$ & $a_{7}$ & $a_{7}$ & $a_{2}$ & $a_{2}$ & $\vdots$ & $a_{2}$ & $a_{2}$ & $\vdots$ & $\vdots$ & $\vdots$ & $\vdots$ & $\vdots$ \\
$\vdots$ & $a_{8}$ & $a_{9}$ & $\vdots$ & $a_{8}$ & $a_{9}$ & $a_{1}$ & $a_{3}$ & $\vdots$ & $a_{1}$ & $a_{3}$ & $\vdots$ & $\vdots$ & $\vdots$ & $\vdots$ & $\vdots$ \\
$\vdots$ & $\vdots$ & $\vdots$ & $\vdots$ & $\vdots$ & $\vdots$ & $\vdots$ & $\vdots$ & $\vdots$ & $\vdots$ & $\vdots$ & $\vdots$ & $\vdots$ & $\vdots$ & $\vdots$ & $\vdots$
\end{tabular}

Table 1: Set of preferences with $a_{5}$ at the top satisfying the richness property

Definition 2.4. An RSCF $\varphi$ is unanimous if $\varphi_{a}\left(P_{N}\right)=1$ for all $a \in A$ and all $P_{N} \in\left(\mathcal{S}^{a}\right)^{N}$.

Definition 2.5. An RSCF $\varphi$ is strategy-proof if for all $i \in N$, all $P_{N} \in \mathcal{S}^{N}$, all $P_{i}^{\prime} \in \mathcal{S}$, and all $x \in A$,

$$
\varphi_{U\left(x, P_{i}\right)}\left(P_{i}, P_{-i}\right) \geq \varphi_{U\left(x, P_{i}\right)}\left(P_{i}^{\prime}, P_{-i}\right) .
$$

For later reference we include the following observation.

Remark 2.1. Let $L, L^{\prime} \in \triangle A$ and let $P \in \mathbb{L}(A)$. Suppose $L_{U(x, P)}=L_{U(x, P)}^{\prime}$ for all $x \in A$. Then $L=L^{\prime}$.

Two profiles $P_{N}, P_{N}^{\prime} \in \mathcal{S}^{N}$ are tops-equivalent if $t\left(P_{i}\right)=t\left(P_{i}^{\prime}\right)$ for all $i \in N$.

Definition 2.6. An RSCF $\varphi$ is tops-only if $\varphi\left(P_{N}\right)=\varphi\left(P_{N}^{\prime}\right)$ for all tops-equivalent $P_{N}, P_{N}^{\prime} \in$ $\mathcal{S}^{N}$.

Definition 2.7. An RSCF $\varphi$ is uncompromising if $\varphi_{d}\left(P_{N}\right)=\varphi_{d}\left(P_{i}^{\prime}, P_{-i}\right)$ for all $i \in N$, all $P_{N} \in \mathcal{S}^{N}$, all $P_{i}^{\prime} \in \mathcal{S}$ and all $d \in A$ such that $d \notin\left[t\left(P_{i}\right), t\left(P_{i}^{\prime}\right)\right]$.

Note that an uncompromising RSCF is tops-only.

\section{Unanimity, strategy-proofness, and uncompromising- ness}

The aim of this section is to prove that on a rich single-peaked domain, unanimity and strategy-proofness of an RSCF imply uncompromisingness.

We introduce the following pieces of notation. For $P \in \mathbb{L}(A)$ and $B \subseteq A,\left.P\right|_{B} \in \mathbb{L}(B)$ is the restriction of $P$ to $B$, that is: for all $a, b \in B,\left.a P\right|_{B} b$ if and only if $a P b$. For $X, Y \subseteq A$ and $P \in \mathbb{L}(A)$, we write $X P Y$ to mean $x P y$ for all $x \in X$ and $y \in Y$. For $P_{N} \in \mathbb{L}(A)^{N}, S \subseteq N$ and $i \in N, P_{-S}$ and $P_{-i}$ denote the restrictions of $P_{N}$ to $N \backslash S$ and $N \backslash\{i\}$, respectively.

The announced result is the following.

Theorem 3.1. Let $\mathcal{S}$ be a rich single-peaked domain, and let $\varphi: \mathcal{S}^{N} \rightarrow \triangle A$ be a unanimous and strategy-proof $R S C F$. Then $\varphi$ is uncompromising.

The proof of this theorem is rather lengthy and involves a number of lemmas. 


\subsection{Proof of Theorem 3.1}

We first prove the following lemma.

Lemma 3.1. Let $\varphi: \mathcal{S}^{N} \rightarrow \triangle A$ be a strategy-proof $R S C F$. Let $P_{N} \in \mathcal{S}^{N}, P_{i}^{\prime} \in \mathcal{S}$, and $B, C \subseteq A$ be such that $B P_{i} C, B P_{i}^{\prime} C$, and $\left.P_{i}\right|_{C}=\left.P_{i}^{\prime}\right|_{C}$. Suppose $\varphi_{C}\left(P_{N}\right)=\varphi_{C}\left(P_{i}^{\prime}, P_{-i}\right)$ and $\varphi_{a}\left(P_{N}\right)=\varphi_{a}\left(P_{i}^{\prime}, P_{-i}\right)$ for all $a \notin B \cup C$. Then $\varphi_{a}\left(P_{N}\right)=\varphi_{a}\left(P_{i}^{\prime}, P_{-i}\right)$ for all $a \in C$.

Proof. First note that since $\varphi_{C}\left(P_{N}\right)=\varphi_{C}\left(P_{i}^{\prime}, P_{-i}\right)$ and $\varphi_{a}\left(P_{N}\right)=\varphi_{a}\left(P_{i}^{\prime}, P_{-i}\right)$ for all $a \notin$ $B \cup C, \varphi_{B}\left(P_{N}\right)=\varphi_{B}\left(P_{i}^{\prime}, P_{-i}\right)$. Suppose $b \in C$ is such that $\varphi_{b}\left(P_{N}\right) \neq \varphi_{b}\left(P_{i}^{\prime}, P_{-i}\right)$ and $\varphi_{a}\left(P_{N}\right)=\varphi_{a}\left(P_{i}^{\prime}, P_{-i}\right)$ for all $a \in C$ with $a P_{i} b$. In other words, $b$ is the maximal element of $C$ according to $P_{i}$ that violates the assertion of the lemma. Without loss of generality, assume that $\varphi_{b}\left(P_{N}\right)<\varphi_{b}\left(P_{i}^{\prime}, P_{-i}\right)$. This, together with the facts that $B P_{i} C, \varphi_{B}\left(P_{N}\right)=\varphi_{B}\left(P_{i}^{\prime}, P_{-i}\right)$, and $\varphi_{a}\left(P_{N}\right)=\varphi_{a}\left(P_{i}^{\prime}, P_{-i}\right)$ for all $a \notin B$ with $a P_{i} b$, implies $\varphi_{U\left(b, P_{i}\right)}\left(P_{N}\right)<\varphi_{U\left(b, P_{i}\right)}\left(P_{i}^{\prime}, P_{-i}\right)$. This means that agent $i$ manipulates at $P_{N}$ via $P_{i}^{\prime}$, which is a contradiction.

We prove the theorem by induction on the number of agents. If $|N|=1$, then uncompromisingness is implied by unanimity. Assume that the theorem holds for all sets with $k<n$ agents. We now prove it for $n$ agents. Let $N^{*}=N \backslash\{1\}$. Define the RSCF $g: \mathcal{S}^{N^{*}} \rightarrow \triangle A$ for $N^{*}$ as follows: for all $P_{N^{*}}=\left(P_{2}, P_{3}, \ldots, P_{n}\right) \in \mathcal{S}^{N^{*}}$,

$$
g\left(P_{2}, P_{3}, \ldots, P_{n}\right)=\varphi\left(P_{2}, P_{2}, P_{3}, P_{4}, \ldots, P_{n}\right) .
$$

Evidently, $g$ is a well-defined RSCF satisfying unanimity and strategy-proofness (see Lemma 3 in Sen (2011) for a detailed argument). Hence, by the induction hypothesis, $g$ satisfies uncompromisingness.

Lemma 3.2. Let $P_{N}, P_{N}^{\prime} \in \mathcal{S}^{N}$ be two tops-equivalent profiles such that $t\left(P_{1}\right)=t\left(P_{2}\right)$. Then $\varphi\left(P_{N}\right)=\varphi\left(P_{N}^{\prime}\right)$.

Proof. Note that since $g$ is uncompromising, $g$ satisfies tops-onlyness, hence $g\left(P_{1}, P_{-\{1,2\}}\right)=$ $g\left(P_{2}, P_{-\{1,2\}}\right)$, and therefore $\varphi\left(P_{1}, P_{1}, P_{-\{1,2\}}\right)=\varphi\left(P_{2}, P_{2}, P_{-\{1,2\}}\right)$. We show that $\varphi\left(P_{1}, P_{2}\right.$, $\left.P_{-\{1,2\}}\right)=\varphi\left(P_{1}, P_{1}, P_{-\{1,2\}}\right)$. Using strategy-proofness of $\varphi$ for agent 2 , we have $\varphi_{U\left(x, P_{1}\right)}\left(P_{1}, P_{1}\right.$, $\left.P_{-\{1,2\}}\right) \geq \varphi_{U\left(x, P_{1}\right)}\left(P_{1}, P_{2}, P_{-\{1,2\}}\right)$ for all $x \in A$, and using strategy-proofness of $\varphi$ for agent 1 , we have $\varphi_{U\left(x, P_{1}\right)}\left(P_{1}, P_{2}, P_{-\{1,2\}}\right) \geq \varphi_{U\left(x, P_{1}\right)}\left(P_{2}, P_{2}, P_{-\{1,2\}}\right)$ for all $x \in A$. Since $\varphi\left(P_{1}, P_{1}, P_{-\{1,2\}}\right)=\varphi\left(P_{2}, P_{2}, P_{-\{1,2\}}\right)$, it follows from Remark 2.1 that $\varphi\left(P_{1}, P_{1}, P_{-\{1,2\}}\right)=$ $\varphi\left(P_{1}, P_{2}, P_{-\{1,2\}}\right)$. Using a similar argument, we have $\varphi\left(P_{1}^{\prime}, P_{1}^{\prime}, P_{-\{1,2\}}^{\prime}\right)=\varphi\left(P_{1}^{\prime}, P_{2}^{\prime}, P_{-\{1,2\}}^{\prime}\right)$. Because $g$ is tops-only and $P_{N}, P_{N}^{\prime}$ are tops-equivalent, we have $g\left(P_{1}, P_{-\{1,2\}}\right)=g\left(P_{1}^{\prime}, P_{-\{1,2\}}^{\prime}\right)$. This means $\varphi\left(P_{1}, P_{1}, P_{-\{1,2\}}\right)=\varphi\left(P_{1}^{\prime}, P_{1}^{\prime}, P_{-\{1,2\}}^{\prime}\right)$, and hence $\varphi\left(P_{1}, P_{2}, P_{-\{1,2\}}\right)=\varphi\left(P_{1}^{\prime}, P_{2}^{\prime}\right.$, $\left.P_{-\{1,2\}}^{\prime}\right)$.

Lemma 3.3. Let $P_{N}, P_{N}^{\prime} \in \mathcal{S}^{N}$ be such that $t\left(P_{1}\right)=t\left(P_{2}\right), t\left(P_{1}^{\prime}\right)=t\left(P_{2}^{\prime}\right)$, and $t\left(P_{i}\right)=t\left(P_{i}^{\prime}\right)$ for all $i \neq 1,2$. Then $\varphi_{c}\left(P_{N}\right)=\varphi_{c}\left(P_{N}^{\prime}\right)$ for all $c \notin\left[t\left(P_{1}\right), t\left(P_{2}\right)\right]$.

Proof. Let $c \notin\left[t\left(P_{1}\right), t\left(P_{2}\right)\right]$. Then

$$
\begin{aligned}
\varphi_{c}\left(P_{1}, P_{2}, P_{-\{1,2]\}}\right) & =\varphi_{c}\left(P_{1}, P_{1}, P_{-\{1,2]\}}\right) \\
& =g_{c}\left(P_{1}, P_{-\{1,2]\}}\right) \\
& =g_{c}\left(P_{1}^{\prime}, P_{-\{1,2]\}}\right) \\
& =\varphi_{c}\left(P_{1}^{\prime}, P_{1}^{\prime}, P_{-\{1,2]\}}\right) \\
& =\varphi_{c}\left(P_{1}^{\prime}, P_{2}^{\prime}, P_{-\{1,2]\}}\right)
\end{aligned}
$$


where the first and last equalities follow from Lemma 3.2, and the third follows since $g$ is uncompromising.

Lemma 3.4. Let $P_{N}, P_{N}^{\prime} \in \mathcal{S}^{N}$ and $a, b \in A$ such that $P_{1}, P_{2}, P_{1}^{\prime} \in \mathcal{S}^{a}, P_{2}^{\prime} \in \mathcal{S}^{b}$, and $t\left(P_{i}\right)=t\left(P_{i}^{\prime}\right)$ for all $i \neq 1,2$. Then $\varphi_{c}\left(P_{N}\right)=\varphi_{c}\left(P_{N}^{\prime}\right)$ for all $c \notin U\left(b, P_{1}^{\prime}\right) \cap U\left(a, P_{2}^{\prime}\right)$.

Proof. By Lemma 3.2, $\varphi\left(P_{1}, P_{2}, P_{-\{1,2\}}\right)=\varphi\left(P_{1}^{\prime}, P_{1}^{\prime}, P_{-\{1,2\}}^{\prime}\right)$. Hence, it suffices to show that $\varphi_{c}\left(P_{1}^{\prime}, P_{1}^{\prime}, P_{-\{1,2\}}^{\prime}\right)=\varphi_{c}\left(P_{1}^{\prime}, P_{2}^{\prime}, P_{-\{1,2\}}^{\prime}\right)$ for $c \notin U\left(b, P_{1}^{\prime}\right) \cap U\left(a, P_{2}^{\prime}\right)$. We prove this for $c \notin U\left(b, P_{1}^{\prime}\right)$, the proof for $c \notin U\left(a, P_{2}^{\prime}\right)$ is analogous.

Take $c \notin U\left(b, P_{1}^{\prime}\right)$. By strategy-proofness of $\varphi$,

$$
\varphi_{U\left(c, P_{1}^{\prime}\right)}\left(P_{1}^{\prime}, P_{1}^{\prime}, P_{-\{1,2\}}^{\prime}\right) \geq \varphi_{U\left(c, P_{1}^{\prime}\right)}\left(P_{1}^{\prime}, P_{2}^{\prime}, P_{-\{1,2\}}^{\prime}\right) \geq \varphi_{U\left(c, P_{1}^{\prime}\right)}\left(P_{2}^{\prime}, P_{2}^{\prime}, P_{-\{1,2\}}^{\prime}\right) .
$$

Moreover, by Lemma 3.3, $\varphi_{x}\left(P_{1}^{\prime}, P_{1}^{\prime}, P_{-\{1,2\}}^{\prime}\right)=\varphi_{x}\left(P_{2}^{\prime}, P_{2}^{\prime}, P_{-\{1,2\}}^{\prime}\right)$ for all $x \notin[a, b]$, and hence $\varphi_{B}\left(P_{1}^{\prime}, P_{1}^{\prime}, P_{-\{1,2\}}^{\prime}\right)=\varphi_{B}\left(P_{2}^{\prime}, P_{2}^{\prime}, P_{-\{1,2\}}^{\prime}\right)$ for all $B \subseteq A$ such that $[a, b] \subseteq B$. Since $c \notin U\left(b, P_{1}^{\prime}\right)$ and $t\left(P_{1}^{\prime}\right)=a$, by definition of a single-peaked domain, we have $[a, b] \subseteq U\left(c, P_{1}^{\prime}\right)$, and hence

$$
\varphi_{U\left(c, P_{1}^{\prime}\right)}\left(P_{1}^{\prime}, P_{1}^{\prime}, P_{-\{1,2\}}^{\prime}\right)=\varphi_{U\left(c, P_{1}^{\prime}\right)}\left(P_{1}^{\prime}, P_{2}^{\prime}, P_{-\{1,2\}}^{\prime}\right) .
$$

Let $d$ be the alternative that ranks just above $c$ in $P_{1}^{\prime}$, i.e., $P_{1}^{\prime}=\cdots d c \cdots$. Then, $[a, b] \subseteq$ $U\left(d, P_{1}^{\prime}\right)$, and hence

$$
\varphi_{U\left(d, P_{1}^{\prime}\right)}\left(P_{1}^{\prime}, P_{1}^{\prime}, P_{-\{1,2\}}^{\prime}\right)=\varphi_{U\left(d, P_{1}^{\prime}\right)}\left(P_{1}^{\prime}, P_{2}^{\prime}, P_{-\{1,2\}}^{\prime}\right) .
$$

Subtracting (2) from (1), we have $\varphi_{c}\left(P_{1}^{\prime}, P_{1}^{\prime}, P_{-\{1,2\}}^{\prime}\right)=\varphi_{c}\left(P_{1}^{\prime}, P_{2}^{\prime}, P_{-\{1,2\}}^{\prime}\right)$, which completes the proof of the lemma.

Lemma 3.5. The RSCF $\varphi$ satisfies the following properties:

(i) $\varphi_{d}\left(P_{N}\right)=\varphi_{d}\left(P_{2}^{\prime}, P_{-2}\right)$ for all $d \notin\left[t\left(P_{1}\right), t\left(P_{2}^{\prime}\right)\right]$, all $P_{N} \in \mathcal{S}^{n}$ with $P_{1}=P_{2}$, and all $P_{2}^{\prime} \in \mathcal{S}$, and

(ii) $\varphi$ is tops-only. ${ }^{4}$

Proof. For $k \geq 0$, let

$$
\mathcal{P}_{k}=\left\{P_{N} \in \mathcal{S}^{N}||\left(t\left(P_{1}\right), t\left(P_{2}\right)\right) \cap A_{J} \mid \leq k\right\} .
$$

Note that $\mathcal{P}_{k}=\mathcal{S}^{n}$ when $k$ is equal to the length of the maximal path in the tree minus two. We prove this lemma by induction on $k$.

Base case. Take $P_{N},\left(P_{2}^{\prime}, P_{-2}\right) \in \mathcal{P}_{0}$. Since $\left|\left(t\left(P_{1}\right), t\left(P_{2}^{\prime}\right)\right) \cap A_{J}\right|=0$, by the definition of single-peakedness, $U\left(t\left(P_{2}^{\prime}\right), P_{1}\right) \cap U\left(t\left(P_{1}\right), P_{2}^{\prime}\right)=\left[t\left(P_{1}\right), t\left(P_{2}^{\prime}\right)\right]$. Hence, by Lemma 3.4 ,

$$
\varphi_{d}\left(P_{N}\right)=\varphi_{d}\left(P_{2}^{\prime}, P_{-2}\right) \forall d \notin\left[t\left(P_{1}\right), t\left(P_{2}^{\prime}\right)\right] .
$$

This proves condition (i) for the base case.

Next, we show condition (ii) for the base case, that is, $\varphi\left(P_{N}\right)=\varphi\left(P_{N}^{\prime}\right)$ for tops-equivalent profiles $P_{N}, P_{N}^{\prime} \in \mathcal{P}_{0}$. It is enough to show that $\varphi\left(P_{N}\right)=\varphi\left(P_{1}^{\prime}, P_{2}, P_{-\{1,2\}}^{\prime}\right)$, since then, by a

\footnotetext{
${ }^{4}$ Chatterji and Zeng (2015) provide a sufficient condition for a domain to be tops-only for RSCFs. However, our domain does not satisfy that condition.
} 
symmetric argument, we can derive $\varphi\left(P_{1}^{\prime}, P_{2}, P_{-\{1,2\}}^{\prime}\right)=\varphi\left(P_{N}^{\prime}\right)$, and combining the two we can conclude $\varphi\left(P_{N}\right)=\varphi\left(P_{N}^{\prime}\right)$.

By Lemma 3.2,

$$
\varphi\left(P_{1}, P_{1}, P_{-\{1,2\}}\right)=\varphi\left(P_{1}^{\prime}, P_{1}^{\prime}, P_{-\{1,2\}}^{\prime}\right) .
$$

Using a similar argument as for the derivation of (3), we have

$$
\begin{gathered}
\varphi_{d}\left(P_{1}, P_{1}, P_{-\{1,2\}}\right)=\varphi_{d}\left(P_{N}\right) \quad \text { for all } d \notin\left[t\left(P_{1}\right), t\left(P_{2}\right)\right], \text { and } \\
\varphi_{d}\left(P_{1}^{\prime}, P_{1}^{\prime}, P_{-\{1,2\}}^{\prime}\right)=\varphi_{d}\left(P_{1}^{\prime}, P_{2}, P_{-2}^{\prime}\right) \quad \text { for all } d \notin\left[t\left(P_{1}^{\prime}\right), t\left(P_{2}\right)\right] .
\end{gathered}
$$

As $t\left(P_{1}\right)=t\left(P_{1}^{\prime}\right)$, combining (4), (5), and (6), we have $\varphi_{d}\left(P_{N}\right)=\varphi_{d}\left(P_{1}^{\prime}, P_{2}, P_{-2}^{\prime}\right)$ for all $d \notin\left[t\left(P_{1}\right), t\left(P_{2}\right)\right]$. Moreover, as $t\left(P_{1}\right)=t\left(P_{1}^{\prime}\right)$, by single-peakedness, $\left.P_{1}\right|_{\left[t\left(P_{1}\right), t\left(P_{2}\right)\right]}=$ $\left.P_{1}^{\prime}\right|_{\left[t\left(P_{1}\right), t\left(P_{2}\right)\right]}$. Now, applying Lemma 3.1 with $B=\emptyset$ and $C=\left[t\left(P_{1}\right), t\left(P_{2}\right)\right]$, we have $\varphi\left(P_{N}\right)=\varphi\left(P_{1}^{\prime}, P_{2}, P_{-2}^{\prime}\right)$. This proves condition (ii) for the base case.

Induction step. Take $k>0$. Suppose that $\varphi$ satisfies property (i) in the lemma when $P_{N},\left(P_{2}^{\prime}, P_{-2}\right) \in \mathcal{P}_{k-1}$, and property (ii) when $\varphi$ is restricted to $\mathcal{P}_{k-1}$, that is, $\varphi\left(P_{N}\right)=\varphi\left(P_{N}^{\prime}\right)$ for all tops-equivalent $P_{N}, P_{N}^{\prime} \in \mathcal{P}_{k-1}$.

Take $P_{N},\left(P_{2}^{\prime}, P_{-2}\right) \in \mathcal{P}_{k} \backslash \mathcal{P}_{k-1}$ with $P_{1}=P_{2}$. Let $c \in\left(t\left(P_{1}\right), t\left(P_{2}^{\prime}\right)\right)$ be the junction node such that there is no junction node in $\left(t\left(P_{2}^{\prime}\right), c\right)$. Since $\left|\left(c, t\left(P_{1}\right)\right) \cap A_{J}\right|=k-1$, by the induction hypothesis, we have for all $d \notin\left[t\left(P_{1}\right), c\right]$ and all $\bar{P} \in \mathcal{S}^{c}$,

$$
\varphi_{d}\left(P_{N}\right)=\varphi_{d}\left(\bar{P}, P_{-2}\right) .
$$

Let $X, Y \subseteq A$ be such that $X=\left\{x \mid t\left(P_{1}\right) \in\left(x, t\left(P_{2}^{\prime}\right)\right]\right\}$ and $Y=\left\{y \mid t\left(P_{2}^{\prime}\right) \in\left(y, t\left(P_{1}\right)\right]\right\}$. We prove the following claims.

Claim 1. Let $\bar{P} \in \mathcal{S}^{c}$ and $d \in X \cup Y$. Then $\varphi_{d}\left(\bar{P}, P_{-2}\right)=\varphi_{d}\left(P_{2}^{\prime}, P_{-2}\right)$.

Proof. Note that $x \notin U\left(t\left(P_{1}\right), P_{2}^{\prime}\right)$ for all $x \in X$ and $y \notin U\left(t\left(P_{2}^{\prime}\right), P_{1}\right)$ for all $y \in Y$. Thus, by Lemma 3.4, $\varphi_{d}\left(P_{N}\right)=\varphi_{d}\left(P_{2}^{\prime}, P_{-2}\right)$. Since $d \notin\left[t\left(P_{1}\right), c\right]$, by $(7), \varphi_{d}\left(P_{N}\right)=\varphi_{d}\left(\bar{P}, P_{-2}\right)$. Hence Claim 1 follows.

Claim 2. Let $\hat{P} \in \mathcal{S}^{c}$. Then $\varphi_{\left[t\left(P_{2}^{\prime}\right), c\right]}\left(P_{2}^{\prime}, P_{-2}\right)=\varphi_{\left[t\left(P_{2}^{\prime}\right), c\right]}\left(\hat{P}, P_{-2}\right)$.

Proof. Let $b \in A_{L}$ be such that $t\left(P_{2}^{\prime}\right) \in[c, b]$. By the richness property, there exists $\bar{P} \in \mathcal{S}^{c}$ such that $\bar{P}=[c, b] \cdots$. By strategy-proofness,

$$
\varphi_{[c, b]}\left(\bar{P}, P_{-2}\right) \geq \varphi_{[c, b]}\left(P_{2}^{\prime}, P_{-2}\right) .
$$

Take $d \in\left(t\left(P_{2}^{\prime}\right), b\right]$. Because $\left[t\left(P_{1}\right), t\left(P_{2}^{\prime}\right)\right] \cap\left(t\left(P_{2}^{\prime}\right), b\right]=\emptyset$, we have $t\left(P_{2}^{\prime}\right) \in\left(d, t\left(P_{1}\right)\right]$ which in turn implies $d \in Y$. Therefore, by Claim $1, \varphi_{d}\left(\bar{P}, P_{-2}\right)=\varphi_{d}\left(P_{2}^{\prime}, P_{-2}\right)$, and hence

$$
\varphi_{\left(t\left(P_{2}^{\prime}\right), b\right]}\left(\bar{P}, P_{-2}\right)=\varphi_{\left(t\left(P_{2}^{\prime}\right), b\right]}\left(P_{2}^{\prime}, P_{-2}\right)
$$

By subtracting (9) from (8), we have

$$
\varphi_{\left[c, t\left(P_{2}^{\prime}\right)\right]}\left(\bar{P}, P_{-2}\right) \geq \varphi_{\left[c, t\left(P_{2}^{\prime}\right)\right]}\left(P_{2}^{\prime}, P_{-2}\right) .
$$

We next show that $\varphi_{\left[c, t\left(P_{2}^{\prime}\right)\right]}\left(P_{2}^{\prime}, P_{-2}\right) \geq \varphi_{\left[c, t\left(P_{2}^{\prime}\right)\right]}\left(\bar{P}, P_{-2}\right)$. By strategy-proofness,

$$
\varphi_{U\left(c, P_{2}^{\prime}\right)}\left(P_{2}^{\prime}, P_{-2}\right) \geq \varphi_{U\left(c, P_{2}^{\prime}\right)}\left(\bar{P}, P_{-2}\right) .
$$


Take $d \in U\left(c, P_{2}^{\prime}\right) \backslash\left[c, t\left(P_{2}^{\prime}\right)\right]$. Using similar arguments as for the derivation of (9), we have

$$
\varphi_{U\left(c, P_{2}^{\prime}\right) \backslash\left[c, t\left(P_{2}^{\prime}\right)\right]}\left(P_{2}^{\prime}, P_{-2}\right)=\varphi_{U\left(c, P_{2}^{\prime}\right) \backslash\left[c, t\left(P_{2}^{\prime}\right)\right]}\left(\bar{P}, P_{-2}\right) .
$$

By subtracting (12) from (11),

$$
\varphi_{\left[c, t\left(P_{2}^{\prime}\right)\right]}\left(P_{2}^{\prime}, P_{-2}\right) \geq \varphi_{\left[c, t\left(P_{2}^{\prime}\right)\right]}\left(\bar{P}, P_{-2}\right) .
$$

Combining (10) and (13), we have $\varphi_{\left[c, t\left(P_{2}^{\prime}\right)\right]}\left(P_{2}^{\prime}, P_{-2}\right)=\varphi_{\left[c, t\left(P_{2}^{\prime}\right)\right]}\left(\bar{P}^{c}, P_{-2}\right)$. Now Claim 2 follows since $\varphi_{\left[t\left(P_{2}^{\prime}\right), c\right]}\left(\hat{P}, P_{-2}\right)=\varphi_{\left[t\left(P_{2}^{\prime}\right), c\right]}\left(\bar{P}, P_{-2}\right)$ by $(7)$.

Claim 3. Let $b \in A_{L}$ such that $t\left(P_{2}^{\prime}\right) \notin[c, b]$. Let $z \in(c, b]$ and $\hat{P} \in \mathcal{S}^{c}$. Then $\varphi_{(c, z]}\left(\hat{P}, P_{-2}\right) \geq \varphi_{(c, z]}\left(P_{2}^{\prime}, P_{-2}\right)$.

Proof. Take $\bar{b} \in A_{L}$ such that $t\left(P_{2}^{\prime}\right) \in[c, \bar{b}]$ and take $\bar{P}=[c, \bar{b}] \cdots(c, b] \cdots$ such that for all $a \in A$ with $c \in[a, \bar{b}] \cap[a, b]$, we have $b \bar{P} a$. Since $c \in A_{J}$ and $\bar{b}, b \in A_{L}$, such a preference exists by richness of $\mathcal{S}$. By strategy-proofness, we have for all $z \in(c, b]$,

$$
\varphi_{U(z, \bar{P})}\left(\bar{P}, P_{-2}\right) \geq \varphi_{U(z, \bar{P})}\left(P_{2}^{\prime}, P_{-2}\right) .
$$

By the definition of $\bar{P}, d \in Y$ for all $d \in U(z, \bar{P}) \backslash\left[t\left(P_{2}^{\prime}\right), z\right]$. Therefore, by Claim 1 ,

$$
\varphi_{U(z, \bar{P}) \backslash\left[t\left(P_{2}^{\prime}\right), z\right]}\left(\bar{P}, P_{-2}\right)=\varphi_{U(z, \bar{P}) \backslash\left[t\left(P_{2}^{\prime}\right), z\right]}\left(P_{2}^{\prime}, P_{-2}\right) .
$$

Subtracting (15) from (14), we obtain $\varphi_{\left[t\left(P_{2}^{\prime}\right), z\right]}\left(\bar{P}, P_{-2}\right) \geq \varphi_{\left[t\left(P_{2}^{\prime}\right), z\right]}\left(P_{2}^{\prime}, P_{-2}\right)$. Since $\left[t\left(P_{2}^{\prime}\right), z\right]=$ $\left[t\left(P_{2}^{\prime}\right), c\right] \cup(c, z]$, by Claim 2 we have

$$
\varphi_{(c, z]}\left(\bar{P}, P_{-2}\right) \geq \varphi_{(c, z]}\left(P_{2}^{\prime}, P_{-2}\right) .
$$

Now Claim 3 follows since $\varphi_{(c, z]}\left(\bar{P}, P_{-2}\right)=\varphi_{(c, z]}\left(\hat{P}, P_{-2}\right)$ by $(7)$.

Claim 4. $\varphi_{d}\left(\bar{P}, P_{-2}\right)=\varphi_{d}\left(P_{2}^{\prime}, P_{-2}\right)$ for all $d \notin\left[c, r_{1}\left(P_{2}^{\prime}\right)\right]$ and all $\bar{P} \in \mathcal{S}^{c}$.

Proof. Assume for contradiction that there exists $d \notin\left[c, r_{1}\left(P_{2}^{\prime}\right)\right]$ and $\bar{P} \in \mathcal{S}^{c}$ such that $\varphi_{d}\left(\bar{P}, P_{-2}\right) \neq \varphi_{d}\left(P_{2}^{\prime}, P_{-2}\right)$. Without loss of generality, assume that $d$ is the maximal alternative according to $P_{2}^{\prime}$ with this property, i.e., $\varphi_{x}\left(\bar{P}, P_{-2}\right)=\varphi_{x}\left(P_{2}^{\prime}, P_{-2}\right)$ for all $x \notin\left[c, r_{1}\left(P_{2}^{\prime}\right)\right]$ with $x P_{2}^{\prime} d$.

First, we show $c P_{2}^{\prime} d$. Suppose not. As $d \notin\left[c, r_{1}\left(P_{2}^{\prime}\right)\right]$ and $c \in\left[r_{1}\left(P_{1}\right), r_{1}\left(P_{2}^{\prime}\right)\right], d P_{2}^{\prime} c$ implies $d \in Y$. However, by Claim 1 , this means $\varphi_{d}\left(\bar{P}, P_{-2}\right)=\varphi_{d}\left(P_{2}^{\prime}, P_{-2}\right)$, a contradiction. This proves $c P_{2}^{\prime} d$.

Now we complete the proof of the claim. By single-peakedness, $\left[c, r_{1}\left(P_{2}^{\prime}\right)\right] \subseteq U\left(d, P_{2}^{\prime}\right)$. Therefore, by Claim 2 and our assumption on $d, \varphi_{U\left(d, P_{2}^{\prime}\right) \backslash\{d\}}\left(\bar{P}, P_{-2}\right)=\varphi_{U\left(d, P_{2}^{\prime}\right) \backslash\{d\}}\left(P_{2}^{\prime}, P_{-2}\right)$. If $\varphi_{d}\left(\bar{P}, P_{-2}\right)>\varphi_{d}\left(P_{2}^{\prime}, P_{-2}\right)$, then agent 2 manipulates at $\left(P_{2}^{\prime}, P_{-2}\right)$ via $\bar{P}$, a contradiction. So, assume $\varphi_{d}\left(P_{2}^{\prime}, P_{-2}\right)>\varphi_{d}\left(\bar{P}, P_{-2}\right)$. Let $\bar{b}, b^{\prime} \in A_{L}$ with $\bar{b} \neq b^{\prime}$ be such that $t\left(P_{2}^{\prime}\right) \in[c, \bar{b}]$ and $d \in\left[c, b^{\prime}\right]$. Such distinct $\bar{b}$ and $b^{\prime}$ must exist as $d \notin Y$ by Claim 1 , hence $d \notin Y \cup\left[t\left(P_{2}^{\prime}\right), c\right]$. Then, by richness of $\mathcal{S}$, there exists $\hat{P}=[c, \bar{b}] \cdots\left(c, b^{\prime}\right] \cdots$ such that $b^{\prime} \hat{P} x$ for all $x \in A$ with the property that $c \in[x, \bar{b}] \cap\left[x, b^{\prime}\right]$. In view of $(7)$, it follows that $\varphi_{d}\left(P_{2}^{\prime}, P_{-2}\right)>\varphi_{d}\left(\hat{P}, P_{-2}\right)$. By the definition of $\hat{P}$, for all $y \notin\left[c, r_{1}\left(P_{2}^{\prime}\right)\right]$ with $y \hat{P} d$, either $y \in Y$ or $y \in(c, d)$. If $y \in Y$, then by Claim $1, \varphi_{y}\left(P_{2}^{\prime}, P_{-2}\right)=\varphi_{y}\left(\hat{P}, P_{-2}\right)$. On the other hand, if $y \in(c, d)$, then by single-peakedness, $y P_{2}^{\prime} d$. By our assumption on $d$, this means $\varphi_{y}\left(P_{2}^{\prime}, P_{-2}\right)=\varphi_{y}\left(\hat{P}, P_{-2}\right)$. Combining, we have $\varphi_{(c, d]}\left(P_{2}^{\prime}, P_{-2}\right)>\varphi_{(c, d]}\left(\hat{P}^{c}, P_{-2}\right)$, which is a contradiction to Claim 3 . This completes the proof of Claim 4. 
We are now ready to complete the proof of the lemma. Since $\left[t\left(P_{1}\right), t\left(P_{2}^{\prime}\right)\right]=\left[t\left(P_{1}\right), c\right] \cup$ $\left(c, t\left(P_{2}^{\prime}\right)\right]$, by $(7)$ and Claim 4 we have $\varphi_{d}\left(P_{N}\right)=\varphi_{d}\left(P_{2}^{\prime}, P_{-2}\right)$ for all $d \notin\left[t\left(P_{1}\right), t\left(P_{2}^{\prime}\right)\right]$. This proves condition (i) for the induction step. Condition (ii) for the induction step now follows by using same arguments as for the proof of (ii) for the base case.

In order to complete the proof of Theorem 3.1, i.e., of the induction step, it is sufficient to prove the following. Let $a, b \in A$ with $\{a, b\} \in E$, and $i \in N$. Then there exist $P_{i} \in \mathcal{S}^{a}$ and $P_{i}^{\prime} \in \mathcal{S}^{b}$ such that for all $P_{-i} \in \mathcal{S}^{N \backslash\{i\}}$ and all $d \notin\{a, b\}$ we have

$$
\varphi_{d}\left(P_{i}, P_{-i}\right)=\varphi_{d}\left(P_{i}^{\prime}, P_{-i}\right)
$$

Since $\varphi$ is tops-only by Lemma 3.5, uncompromisingness follows by repeated application of (17).

We first show (17) if $i=1$. Let $P_{-1} \in \mathcal{S}^{N \backslash\{1\}}$ and $t\left(P_{2}\right)=c$ for some $c \in A$. Without loss of generality assume $b \in[a, c]$. By richness of $\mathcal{S}$, it contains preferences $P_{1}=a b \cdots$ and $P_{1}^{\prime}=b a \cdots$. By Lemma 3.5, $\varphi_{d}\left(P_{1}, P_{2}, P_{-\{1,2\}}\right)=\varphi_{d}\left(P_{2}, P_{2}, P_{-\{1,2\}}\right)$ for all $d \notin[a, c]$ and $\varphi_{d}\left(P_{1}^{\prime}, P_{2}, P_{-\{1,2\}}\right)=\varphi_{d}\left(P_{2}, P_{2}, P_{-\{1,2\}}\right)$ for all $d \notin[b, c]$. This implies $\varphi_{d}\left(P_{1}, P_{2}, P_{-\{1,2\}}\right)=$ $\varphi_{d}\left(P_{1}^{\prime}, P_{2}, P_{-\{1,2\}}\right)$ for all $d \notin[a, c]$. By strategy-proofness, $\varphi_{\{a, b\}}\left(P_{1}, P_{2}, P_{-\{1,2\}}\right)=\varphi_{\{a, b\}}\left(P_{1}^{\prime}\right.$, $\left.P_{2}, P_{-\{1,2\}}\right)$, and hence $\varphi_{[a, c] \backslash\{a, b\}}\left(P_{1}, P_{2}, P_{-\{1,2\}}\right)=\varphi_{[a, c] \backslash\{a, b\}}\left(P_{1}^{\prime}, P_{2}, P_{-\{1,2\}}\right)$. Since $\{a, b\} \in$ $E, t\left(P_{1}\right)=a$, and $t\left(P_{1}^{\prime}\right)=b$, by single-peakedness we have $\left.P_{1}\right|_{[a, c] \backslash\{a, b\}}=\left.P_{1}^{\prime}\right|_{[a, c] \backslash\{a, b\}}$. By using Lemma 3.1 with $B=\{a, b\}$ and $C=[a, c] \backslash\{a, b\}$, we have $\varphi_{d}\left(P_{1}, P_{2}, P_{-\{1,2\}}\right)=$ $\varphi_{d}\left(P_{1}^{\prime}, P_{2}, P_{-\{1,2\}}\right)$ for all $d \neq a, b$. This proves (17) for agent 1. Using a symmetric argument, (17) can be shown for agent 2 .

Finally, we show (17) for agents $i \in\{3, \ldots, n\}$. It is sufficient to show this for $i=3$. By richness of $\mathcal{S}$, it contains preferences $P_{3}=a b \cdots$ and $P_{3}^{\prime}=b a \cdots$. Let $P_{-3} \in \mathcal{S}^{N \backslash\{3\}}$. By strategy-proofness,

$$
\varphi_{\{a, b\}}\left(P_{1}, P_{2}, P_{3}, P_{-\{1,2,3\}}\right)=\varphi_{\{a, b\}}\left(P_{1}, P_{2}, P_{3}^{\prime}, P_{-\{1,2,3\}}\right) .
$$

Let $t\left(P_{1}\right)=c$ and $t\left(P_{2}\right)=c^{\prime}$. We distinguish the following cases.

CASE 1. $c \in\left[a, c^{\prime}\right]$ or $c^{\prime} \in[a, c]$.

By Lemma 3.5, $\varphi_{d}\left(P_{1}, P_{1}, P_{3}, P_{-\{1,2,3\}}\right)=\varphi_{d}\left(P_{1}, P_{2}, P_{3}, P_{-\{1,2,3\}}\right)$ and $\varphi_{d}\left(P_{1}, P_{1}, P_{3}^{\prime}, P_{-\{1,2,3\}}\right)$ $=\varphi_{d}\left(P_{1}, P_{2}, P_{3}^{\prime}, P_{-\{1,2,3\}}\right)$ for all $d \notin\left[c, c^{\prime}\right]$. Also, by the induction hypothesis, $\varphi_{d}\left(P_{1}, P_{1}, P_{3}\right.$, $\left.P_{-\{1,2,3\}}\right)=\varphi_{d}\left(P_{1}, P_{1}, P_{3}^{\prime}, P_{-\{1,2,3\}}\right)$ for all $d \notin\{a, b\}$. Combining all these, we have for all $d \notin\left[c, c^{\prime}\right] \cup\{a, b\}$,

$$
\varphi_{d}\left(P_{1}, P_{2}, P_{3}, P_{-\{1,2,3\}}\right)=\varphi_{d}\left(P_{1}, P_{2}, P_{3}^{\prime}, P_{-\{1,2,3\}}\right) .
$$

By (19), (18) implies $\varphi_{\left[c, c^{\prime}\right] \backslash\{a, b\}}\left(P_{1}, P_{2}, P_{3}, P_{-\{1,2,3\}}\right)=\varphi_{\left[c, c^{\prime}\right] \backslash\{a, b\}}\left(P_{1}, P_{2}, P_{3}^{\prime}, P_{-\{1,2,3\}}\right)$. Since $\{a, b\}) \in E$, by single-peakedness, both $c \in\left[a, c^{\prime}\right]$ and $c^{\prime} \in[a, c]$ imply $\left.P_{3}\right|_{\left[c, c^{\prime}\right] \backslash\{a, b\}}=$ $\left.P_{3}^{\prime}\right|_{\left[c, c^{\prime}\right] \backslash\{a, b\}}$. Therefore, by applying Lemma 3.1 with $B=\{a, b\}$ and $C=\left[c, c^{\prime}\right] \backslash\{a, b\}$, and by (19), we have $\varphi_{d}\left(P_{1}, P_{2}, P_{3}, P_{-\{1,2,3\}}\right)=\varphi_{d}\left(P_{1}, P_{2}, P_{3}^{\prime}, P_{-\{1,2,3\}}\right)$ for all $d \notin\{a, b\}$, and hence (17) for this case.

CASE 2. $c \notin\left[a, c^{\prime}\right]$ and $c^{\prime} \notin[a, c]$.

Let $x \in\left(c, c^{\prime}\right)$ such that $x \in(c, a] \cap\left(c^{\prime}, a\right]$. Let $P^{x} \in \mathcal{S}^{x}$. Then by Case 1 , for all $d \notin\{a, b\}$,

$$
\begin{aligned}
& \varphi_{d}\left(P^{x}, P_{2}, P_{3}, P_{-\{1,2,3\}}\right)=\varphi_{d}\left(P^{x}, P_{2}, P_{3}^{\prime}, P_{-\{1,2,3\}}\right), \text { and } \\
& \varphi_{d}\left(P_{1}, P^{x}, P_{3}, P_{-\{1,2,3\}}\right)=\varphi_{d}\left(P_{1}, P^{x}, P_{3}^{\prime}, P_{-\{1,2,3\}}\right) .
\end{aligned}
$$


By (17) for agents 1 and $2, \varphi_{d}\left(P_{1}, P_{2}, P_{3}, P_{-\{1,2,3\}}\right)=\varphi_{d}\left(P^{x}, P_{2}, P_{3}, P_{-\{1,2,3\}}\right)$ and $\varphi_{d}\left(P_{1}, P_{2}, P_{3}^{\prime}\right.$, $\left.P_{-\{1,2,3\}}\right)=\varphi_{d}\left(P^{x}, P_{2}, P_{3}^{\prime}, P_{-\{1,2,3\}}\right)$ for all $e \notin[x, c]$. By $(20)$, this implies for all $d \notin$ $\{a, b\} \cup[x, c]$,

$$
\varphi_{d}\left(P_{1}, P_{2}, P_{3}, P_{-\{1,2,3\}}\right)=\varphi_{d}\left(P_{1}, P_{2}, P_{3}^{\prime}, P_{-\{1,2,3\}}\right) .
$$

By analogous arguments we obtain for all $d \notin\{a, b\} \cup\left[x, c^{\prime}\right]$,

$$
\varphi_{d}\left(P_{1}, P_{2}, P_{3}, P_{-\{1,2,3\}}\right)=\varphi_{d}\left(P_{1}, P_{2}, P_{3}^{\prime}, P_{-\{1,2,3\}}\right) .
$$

Since $[x, c] \cap\left[x, c^{\prime}\right]=\{x\}$, by (22) and (23), we have for all $d \notin\{a, b, x\}$,

$$
\varphi_{d}\left(P_{1}, P_{2}, P_{3}, P_{-\{1,2,3\}}\right)=\varphi_{d}\left(P_{1}, P_{2}, P_{3}^{\prime}, P_{-\{1,2,3\}}\right) .
$$

If $x=a$, then $(24)$ implies $\varphi_{d}\left(P_{1}, P_{2}, P_{3}, P_{-\{1,2,3\}}\right)=\varphi_{d}\left(P_{1}, P_{2}, P_{3}^{\prime}, P_{-\{1,2,3\}}\right)$ for all $d \notin\{a, b\}$, establishing (17) for agent 3. If $x \neq a$, then by (18) and $(24), \varphi_{d}\left(P_{1}, P_{2}, P_{3}, P_{-\{1,2,3\}}\right)=$ $\varphi_{d}\left(P_{1}, P_{2}, P_{3}^{\prime}, P_{-\{1,2,3\}}\right)$ for all $d \notin\{a, b\}$, again establishing (17) for agent 3 .

Since Cases 1 and 2 are exhaustive, this completes the proof of the theorem.

\section{Characterization of all unanimous and strategy-proof RSCFs}

Throughout, $\mathcal{S}$ is a rich single-peaked domain.

An allocation function is a function $\mu: N \rightarrow A_{L}$, and the set of all allocation functions is denoted by $\mathcal{M}$. In other words, an allocation function assigns each agent to a leaf of the tree.

Definition 4.1. For $a \in A, P_{N} \in \mathcal{S}^{N}$, an allocation function $\mu$ is tree-monotonic with respect to $\left(a, P_{N}\right)$ if for all $i \in N$ and $b \in A_{L}, \mu(i)=b$ implies $t\left(P_{i}\right) \in[a, b]$.

Thus, an allocation function that is tree-monotonic with respect to an alternative $a$ and a preference profile assigns each agent to a leaf such that there is a path from $a$ to that leaf containing the agent's peak.

The set of allocation functions that are tree-monotonic with respect to $\left(a, P_{N}\right)$ is denoted by $\mathcal{M}\left(a, P_{N}\right)$. Clearly, if $P_{N}, P_{N}^{\prime} \in \mathcal{S}^{N}$ are tops-equivalent, then $\mathcal{M}\left(a, P_{N}\right)=\mathcal{M}\left(a, P_{N}^{\prime}\right)$. The following example illustrates Definition 4.1 .

Example 4.1. Consider the tree in Figure 2. Let $N=\{1, \ldots, 6\}$. For the purpose of evaluating a tree-monotonic function, by the preceding remark it is sufficient to represent a profile by its top-alternatives. Consider, then, the profile $P_{N}=\left(a_{1}, a_{3}, a_{4}, a_{4}, a_{5}, a_{7}\right)$. In Table 2, we consider some choices of $a$, and provide the values of a tree-monotonic allocation function $\mu$ with respect to $\left(a, P_{N}\right)$. The values of $\mu(i)$ for $i=1, \ldots, 6$ are shown in the columns. The rest of the table is self-explanatory.

Definition 4.2. A collection of probability distributions $\left(\beta_{\mu}\right)_{\mu \in \mathcal{M}}$ over $A$ is called treemonotonic if it satisfies

(i) for every $b \in A, \beta_{\mu}(b)=1$ if $\mu(i)=b$ for all $i \in N$,

(ii) for all $\mu, \hat{\mu} \in \mathcal{M}$ and $i \in N$ such that $\mu(j)=\hat{\mu}(j)$ for all $j \in N \backslash\{i\}$, 


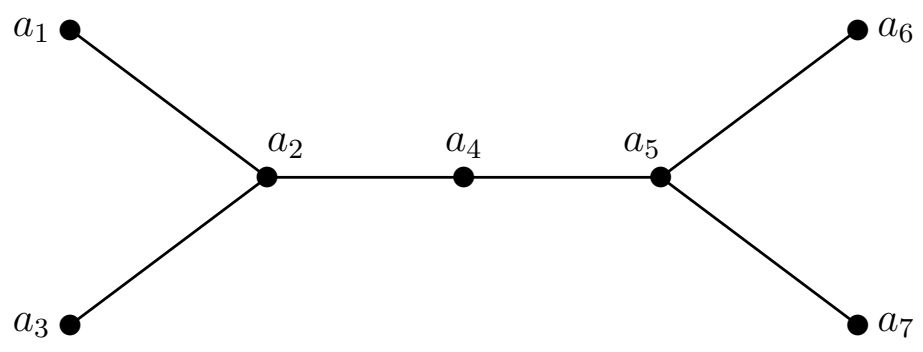

Figure 2: The tree in Example 4.1

\begin{tabular}{c|c|c|c|c|c|c}
$a$ & $\mu(1)$ & $\mu(2)$ & $\mu(3)$ & $\mu(4)$ & $\mu(5)$ & $\mu(6)$ \\
\hline$a_{2}$ & $a_{1}$ & $a_{3}$ & $a_{7}$ & $a_{7}$ & $a_{7}$ & $a_{7}$ \\
$a_{4}$ & $a_{1}$ & $a_{3}$ & $a_{3}$ & $a_{3}$ & $a_{6}$ & $a_{7}$ \\
$a_{6}$ & $a_{1}$ & $a_{3}$ & $a_{3}$ & $a_{3}$ & $a_{7}$ & $a_{7}$
\end{tabular}

Table 2: Illustration of $\mu$

(a) $\beta_{\hat{\mu}}([c, \hat{\mu}(i)]) \geq \beta_{\mu}([c, \hat{\mu}(i)])$ for all $c \in[\mu(i), \hat{\mu}(i)]$,

(b) $\beta_{\mu}(c)=\beta_{\hat{\mu}}(c)$ for all $c \in A \backslash[\mu(i), \hat{\mu}(i)]$.

Part (i) in this definition says that if all agents are assigned to the same leaf then that node gets probability one. Part (ii) says that if an agent $i$ moves from one leaf (at $\mu$ ) to another (at $\hat{\mu}$ ), then probability increases along the path from the former to the latter leaf (part (a)), whereas off this path nothing changes (part (b)). Clearly, the conditions (i), (ii)(a), and (ii)(b), are the counterparts of the conditions of unanimity, strategy-proofness, and uncompromisingness of an RSCF.

The following example illustrates the notion of tree-monotonic probability distributions.

Example 4.2. Consider the tree given in Figure 2. Let $N=\{1,2,3\}$. Consider the probability distributions $\left(\beta_{\mu}\right)_{\mu \in \mathcal{M}}$ given in Table 3 . For simplicity, we assume that the collection $\left(\beta_{\mu}\right)_{\mu \in \mathcal{M}}$ is 'anonymous', which means that the probabilities depend only on the numbers of agents on the leafs. It is left to the reader to verify that $\left(\beta_{\mu}\right)_{\mu \in \mathcal{M}}$ in this table satisfies tree-monotonicity.

\begin{tabular}{c|c||c|c}
$\mu$ & $\beta_{\mu}$ & $\mu$ & $\beta_{\mu}$ \\
\hline$(3,0,0,0)$ & $(1,0,0,0,0,0,0)$ & $(1,0,2,0)$ & $(.1, .3,0, .3, .2, .2,0)$ \\
$(0,3,0,0)$ & $(0,0,1,0,0,0,0)$ & $(0,1,2,0)$ & $(0, .2, .3, .2, .1, .2,0)$ \\
$(0,0,3,0)$ & $(0,0,0,0,0,1,0)$ & $(0,0,2,1)$ & $(0,0,0,0, .7, .2, .1)$ \\
$(0,0,0,3)$ & $(0,0,0,0,0,0,1)$ & $(1,0,0,2)$ & $(.1, .3,0, .3, .2,0, .2)$ \\
$(2,1,0,0)$ & $(.4, .3, .3,0,0,0,0)$ & $(0,1,0,2)$ & $(0, .2, .3, .2, .1,0, .2)$ \\
$(2,0,1,0)$ & $(.4, .2,0, .2, .1, .1,0)$ & $(0,0,1,2)$ & $(0,0,0,0, .7, .1, .2)$ \\
$(2,0,0,1)$ & $(.4, .2,0, .2, .1,0, .1)$ & $(1,1,1,0)$ & $(.1, .2, .3, .2, .1, .1,0)$ \\
$(1,2,0,0)$ & $(.1, .5, .4,0,0,0,0)$ & $(1,1,0,1)$ & $(.1, .2, .3, .2, .1,0, .1)$ \\
$(0,2,1,0)$ & $(0, .2, .4, .2, .1, .1,0)$ & $(1,0,1,1)$ & $(.1, .3,0, .3, .2, .1, .1)$ \\
$(0,2,0,1)$ & $(0, .2, .4, .2, .1,0, .1)$ & $(0,1,1,1)$ & $(0, .2, .3, .2, .1, .1, .1)$
\end{tabular}

Table 3: Example 4.2 
Below we will associate an RSCF with each tree-monotonic collection of probability distributions. As a preparation we need the following lemma.

Lemma 4.1. Let $\left(\beta_{\mu}\right)_{\mu \in \mathcal{M}}$ be a tree-monotonic collection of probability distributions. Let $a \in A, b, c \in A_{L}, P_{N} \in \mathcal{S}^{N}$, and $\mu_{b}, \hat{\mu}_{b}, \mu_{c}, \hat{\mu}_{c} \in \mathcal{M}\left(a, P_{N}\right)$ such that for each $x \in\{b, c\}$ and all $i \in N, \mu_{x}(i)=x$ if and only if $t\left(P_{i}\right) \in[a, x]$ and $\hat{\mu}_{x}(i)=x$ if and only if $t\left(P_{i}\right) \in(a, x]$. Then

$$
\beta_{\mu_{b}}(\pi[a, b])-\beta_{\hat{\mu}_{b}}(\pi(a, b])=\beta_{\mu_{c}}(\pi[a, c])-\beta_{\hat{\mu}_{c}}(\pi(a, c]) \geq 0 .
$$

Proof. First, we show that the left-hand side of (25) does not depend on any particular choice of $\hat{\mu}_{b}$. An analogous argument holds for the right-hand side. Take $\hat{\mu}_{b}^{\prime} \in \mathcal{M}\left(a, P_{N}\right)$ such that for all $i \in N, \hat{\mu}_{x}^{\prime}(i)=x$ if and only if $t\left(P_{i}\right) \in(a, x]$. Then, by condition (ii)(b) in Definition 4.2 we have

$$
\beta_{\hat{\mu}_{x}}((a, x])=\beta_{\hat{\mu}_{x}^{\prime}}((a, x]) .
$$

It is sufficient to prove the lemma for the case where $a \in[b, c]$. Otherwise, there is a $d \in A_{L}$ such that both $a \in[d, b]$ and $a \in[d, c]$. Then, if we show (25) for the pairs of leafs $b, d$ and $c, d,(25)$ follows for the pair $b, c$ by combining the two equations. Thus, we assume $a \in[b, c]$. In view of $(26)$, it is sufficient to show that

$$
\beta_{\mu_{b}}([a, b])-\beta_{\mu_{c}}((a, b])=\beta_{\mu_{c}}([a, c])-\beta_{\mu_{b}}((a, c]) .
$$

By condition (ii)(b) in Definition 4.2 we have

$$
\beta_{\mu_{b}}[b, c]=\beta_{\mu_{c}}[b, c]
$$

Therefore,

$$
\begin{aligned}
\beta_{\mu_{b}}([a, b])-\beta_{\mu_{c}}((a, b]) & =\beta_{\mu_{b}}([b, c])-\beta_{\mu_{b}}((a, c])-\beta_{\mu_{c}}((a, b]) \\
& =\beta_{\mu_{c}}([b, c])-\beta_{\mu_{c}}((a, b])-\beta_{\mu_{b}}((a, c]) \\
& =\beta_{\mu_{c}}([a, c])-\beta_{\mu_{b}}((a, c])
\end{aligned}
$$

where the second equality follows from (27).

Finally, by condition (ii)(a) in Definition 4.2 we have

$$
\beta_{\hat{\mu}_{b}}((a, b]) \leq \beta_{\mu_{b}}((a, b]),
$$

which implies the nonnegativity of the expressions in (25) and completes the proof of the lemma.

Let $B=\left(\beta_{\mu}\right)_{\mu \in \mathcal{M}}$ be a tree-monotonic collection of probability distributions over $A$. We associate a map $\varphi^{B}: \mathcal{S}^{N} \rightarrow \triangle A$ by defining, for each $a \in A$ and $P_{N} \in \mathcal{S}_{N}$,

$$
\varphi_{a}^{B}\left(P_{N}\right)=\beta_{\mu_{b}}([a, b])-\beta_{\hat{\mu}_{b}}((a, b])
$$

for some $b \in A_{L}$ and $\mu_{b}, \hat{\mu}_{b} \in \mathcal{M}\left(a, P_{N}\right)$ such that $\mu_{b}(i)=b$ if and only if $t\left(P_{i}\right) \in[a, b]$ and $\hat{\mu}_{b}(i)=b$ if and only if $t\left(P_{i}\right) \in(a, b]$. In words, the probability assigned by $\varphi^{B}$ to alternative $a$ if the preference profile is $P_{N}$, is determined as follows. Take an arbitrary leaf $b$, take an allocation function where all agents with peaks on the path $[a, b]$ are assigned to $b$, and compute the sum of the associated probabilities assigned to alternatives on the path $[a, b]$. Next, take an allocation function where all agents with peaks on the path $(a, b]$ (but not 
those with peak at $a$ ) are assigned to $b$, and compute the sum of the associated probabilities assigned to alternatives on the path $(a, b]$. The difference between these numbers is the probability assigned to $a$.

Note that by Lemma 4.1, $\varphi^{B}$ is well-defined: it does not depend on the particular choice of $b, \mu_{b}$, or $\hat{\mu}_{b}$. We also have:

Lemma 4.2. $\varphi^{B}$ defined by (28) is an RSCF.

Proof. By Lemma 4.1, $\varphi_{a}^{B}\left(P_{N}\right) \geq 0$ for every $a \in A$ and $P_{N} \in \mathcal{S}^{N}$. We still have to prove that $\sum_{a \in A} \varphi_{a}^{B}\left(P_{N}\right)=1$ for every $P_{N} \in \mathcal{S}^{N}$.

Let $a \in A, b \in A_{L}$, and let $\mu \in \mathcal{M}\left(a, P_{N}\right)$ such that $\mu(i)=b$ if and only if $t\left(P_{i}\right) \in[a, b]$, for all $i \in N$. We claim that $\varphi_{[a, b]}^{B}\left(P_{N}\right)=\beta_{\mu}([a, b])$. To show this, write $[a, b]=\left(a_{1}, \ldots, a_{k}\right)$, where $a=a_{1}$ and $b=a_{k}$. For every $j=1, \ldots, k$ let $\mu_{j}, \hat{\mu}_{j} \in \mathcal{M}\left(a_{j}, P_{N}\right)$ such that for all $i \in N$ we have $\mu(j)=b \Leftrightarrow t\left(P_{i}\right) \in\left[a_{j}, b\right]$ and $\hat{\mu}(j)=b \Leftrightarrow t\left(P_{i}\right) \in\left(a_{j}, b\right]$. Then

$$
\begin{aligned}
\varphi_{[a, b]}^{B}\left(P_{N}\right)= & \beta_{\mu_{1}}\left(\left[a_{1}, b\right]\right)-\beta_{\hat{\mu}_{1}}\left(\left(a_{1}, b\right]\right) \\
& +\beta_{\mu_{2}}\left(\left[a_{2}, b\right]\right)-\beta_{\hat{\mu}_{2}}\left(\left(a_{2}, b\right]\right) \\
& +\beta_{\mu_{3}}\left(\left[a_{3}, b\right]\right)-\beta_{\hat{\mu}_{3}}\left(\left(a_{3}, b\right]\right) \\
& \vdots \\
& +\beta_{\mu_{k}}(\{b\})-\beta_{\hat{\mu}_{k}}(\emptyset) \\
= & \beta_{\mu_{1}}\left(\left[a_{1}, b\right]\right) \\
= & \beta_{\mu}([a, b])
\end{aligned}
$$

where the before last equality follows since $\beta_{\hat{\mu}_{j}}\left(\left(a_{j}, b\right]\right)=\beta_{\hat{\mu}_{j}}\left(\left[a_{j+1}, b\right]\right)=\beta_{\mu_{j+1}}\left(\left[a_{j+1}, b\right]\right)$ for every $j=1, \ldots, k-1$ by condition (ii)(b) in Definition 4.2 , and also the last equality follows from this condition.

Now let $P_{N} \in \mathcal{S}^{N}$ be arbitrary. We partition $A$ into subsets $A^{1}, \ldots, A^{k}$, such that each $A^{\ell}$ is of the form $A^{\ell}=\left[a^{\ell}, \ldots, b^{\ell}\right]$ for some $b^{\ell} \in A_{L}$ (possibly $a^{\ell}=b^{\ell}$ ). We define the allocation function $\mu$ as follows: (i) for each $\ell=1, \ldots, k, \mu^{-1}\left(b^{\ell}\right)=\left\{i \in N \mid t\left(P_{i}\right) \in A^{\ell}\right\}$, and (ii) for each $b \in A_{L} \backslash\left\{b^{1}, \ldots, b^{k}\right\}, \mu^{-1}(b)=\emptyset$ (case (ii) occurs if $b=a^{\ell}$ for some $\ell$ ). Then by condition (ii)(b) in Definition 4.2 , for every $\ell=1, \ldots, k$ there is a $\mu_{\ell} \in \mathcal{M}\left(a^{\ell}, P_{N}\right)$ such that $\beta_{\mu_{\ell}}\left(A^{\ell}\right)=\beta_{\mu}\left(A^{\ell}\right)$. Hence, by the previous part of the proof, $\sum_{a \in A} \varphi_{a}^{B}\left(P_{N}\right)=$ $\sum_{\ell=1}^{k} \beta_{\mu_{\ell}}\left(A^{\ell}\right)=\sum_{\ell=1}^{k} \beta_{\mu}\left(A^{\ell}\right)=\beta_{\mu}(A)=1$.

We call $\varphi^{B}$ a tree-monotonic RSCF. An example of a tree-monotonic RSCF is the following.

Example 4.3. Consider the tree given in Figure 2. Let $N=\{1,2,3\}$. Since a treemonotonic RSCF is tops-only, to evaluate the outcome of such an RSCF at some profile we need to consider only the top alternatives of the agents at that profile. Let $\varphi$ be the tree-monotonic (anonymous) RSCF with respect to $\left(\beta_{\mu}\right)_{\mu \in \mathcal{M}}$ as given in Table 3 . Consider the profile $\left(a_{2}, a_{4}, a_{6}\right)$. The outcome of $\varphi$ at $\left(a_{2}, a_{4}, a_{6}\right)$ is shown in Table 4 . The table is self-explanatory.

Our main result will be that these tree-monotonic RSCFs are exactly the unanimous and strategy-proof RSCFs on our rich single-peaked domain. 


\begin{tabular}{c|c|c|c}
$a$ & $b$ & $\beta_{\mu}(\pi[a, b])-\beta_{\mu^{\prime}}(\pi(a, b])$ & $\varphi_{a}\left(a_{2}, a_{4}, a_{6}\right)$ \\
\hline$a_{1}$ & $a_{1}$ & $\beta_{(0,3,0,0)}\left(\pi\left[a_{1}, a_{1}\right]\right)-\beta_{(0,3,0,0)}\left(\pi\left(a_{1}, a_{1}\right]\right)$ & 0 \\
$a_{2}$ & $a_{1}$ & $\beta_{(1,0,2,0)}\left(\pi\left[a_{2}, a_{1}\right]\right)-\beta_{(0,1,2,0)}\left(\pi\left(a_{2}, a_{1}\right]\right)$ & .4 \\
$a_{3}$ & $a_{1}$ & $\beta_{(1,0,2,0)}\left(\pi\left[a_{3}, a_{1}\right]\right)-\beta_{(1,0,2,0)}\left(\pi\left(a_{3}, a_{1}\right]\right)$ & 0 \\
$a_{4}$ & $a_{1}$ & $\beta_{(2,0,1,0)}\left(\pi\left[a_{4}, a_{1}\right]\right)-\beta_{(1,0,2,0)}\left(\pi\left(a_{4}, a_{1}\right]\right)$ & .4 \\
$a_{5}$ & $a_{1}$ & $\beta_{(2,0,1,0)}\left(\pi\left[a_{5}, a_{1}\right]\right)-\beta_{(2,0,1,0)}\left(\pi\left(a_{5}, a_{1}\right]\right)$ & .1 \\
$a_{6}$ & $a_{6}$ & $\beta_{(2,0,1,0)}\left(\pi\left[a_{6}, a_{6}\right]\right)-\beta_{(2,0,0,1)}\left(\pi\left(a_{6}, a_{6}\right]\right)$ & .1 \\
$a_{7}$ & $a_{6}$ & $\beta_{(2,0,1,0)}\left(\pi\left[a_{7}, a_{6}\right]\right)-\beta_{(2,0,1,0)}\left(\pi\left(a_{7}, a_{6}\right]\right)$ & 0
\end{tabular}

Table 4: An example showing the values of $\varphi_{a}\left(P_{N}\right)$

Lemma 4.3. Let $B=\left(\beta_{\mu}\right)_{\mu \in \mathcal{M}}$ be a tree-monotonic collection of probability distributions over $A$. Then $\varphi^{B}$ is unanimous and strategy-proof.

Proof. Unanimity follows directly from the definition of $\varphi$. In this proof we write $\varphi$ instead of $\varphi^{B}$ and first show that $\varphi$ is uncompromising. Let $P_{N} \in \mathcal{S}^{N}, i \in N, P_{i}^{\prime} \in \mathcal{S}$, and $d \in A \backslash\left[t\left(P_{i}\right), t\left(P_{i}^{\prime}\right)\right]$. Take $b \in A_{L}$ such that $[d, b] \cap\left[t\left(P_{i}\right), t\left(P_{i}^{\prime}\right)\right]=\emptyset$. Then, by definition of $\varphi$, in particular $(28)$, we obtain $\varphi_{d}\left(P_{N}\right)=\varphi_{d}\left(P_{-i}, P_{i}^{\prime}\right)$. This shows that $\varphi$ is uncompromising.

In order to prove strategy-proofness, assume for contradiction that there exists $i \in N$, $P_{N} \in \mathcal{S}^{N}$, and $P_{i}^{\prime} \in \mathcal{S}$ such that $\varphi_{U\left(c, P_{i}\right)}\left(P_{N}\right)<\varphi_{U\left(c, P_{i}\right)}\left(P_{i}^{\prime}, P_{-i}\right)$ for some $c \in A$. Since $\varphi$ is uncompromising, we have $\varphi_{z}\left(P_{N}\right)=\varphi_{z}\left(P_{i}^{\prime}, P_{-i}\right)$ for all $z \notin\left[t\left(P_{i}\right), t\left(P_{i}^{\prime}\right)\right]$. Therefore, we may assume without loss of generality that $c \in\left[t\left(P_{i}\right), t\left(P_{i}^{\prime}\right)\right)$ and

$$
\varphi_{\pi\left[r_{1}\left(P_{i}\right), c\right]}\left(P_{N}\right)<\varphi_{\pi\left[r_{1}\left(P_{i}\right), c\right]}\left(P_{i}^{\prime}, P_{-i}\right) .
$$

Let $d$ appear just after $c$ along the path $\left[t\left(P_{i}\right), t\left(P_{i}^{\prime}\right)\right]$. Let $P^{c} \in \mathcal{S}^{c}$ and $P^{d} \in \mathcal{S}^{d}$. By uncompromisingness, $\varphi_{\left[t\left(P_{i}\right), c\right]}\left(P_{N}\right)=\varphi_{\left[t\left(P_{i}\right), c\right]}\left(P^{c}, P_{-i}\right)$ and $\varphi_{\left[t\left(P_{i}\right), c\right]}\left(P^{d}, P_{-i}\right)=\varphi_{\left[t\left(P_{i}\right), c\right]}\left(P_{i}^{\prime}, P_{-i}\right)$. By (29), this yields $\varphi_{\left[t\left(P_{i}\right), c\right]}\left(P^{c}, P_{-i}\right)<\varphi_{\left[t\left(P_{i}\right), c\right]}\left(P^{d}, P_{-i}\right)$. Since by uncompromisingness $\varphi_{z}\left(P^{c}, P_{-i}\right)=\varphi_{z}\left(P^{d}, P_{-i}\right)$ for all $z \notin\{c, d\}$, this implies

$$
\varphi_{c}\left(P^{c}, P_{-i}\right)<\varphi_{c}\left(P^{d}, P_{-i}\right) .
$$

Now take $b, b^{\prime} \in A_{L}$ such that $\{c, d\} \subseteq\left[b, b^{\prime}\right]$ and $d \notin[b, c]$. By (28),

$$
\varphi_{c}\left(P^{c}, P_{-i}\right)=\beta_{\mu_{b}}([c, b])-\beta_{\hat{\mu}_{b}}((c, b])
$$

where $\mu_{b}, \hat{\mu}_{b} \in \mathcal{M}\left(c,\left(P^{c}, P_{-i}\right)\right)$ are such that $\mu_{b}(j)=b$ if and only if $t\left(P_{j}\right) \in[c, b]$ and $\hat{\mu}_{b}(j)=b$ if and only if $t\left(P_{j}\right) \in(c, b]$ for all $j \in N$. Let $\mu_{b}^{\prime}$ be such that $\mu_{b}^{\prime}(j)=\mu(j)$ for all $j \in N \backslash\{i\}$ and $\mu_{b}^{\prime}(i)=b^{\prime}$; and let $\hat{\mu}_{b}^{\prime}=\hat{\mu}_{b}$. Note that $\mu_{b}^{\prime}, \hat{\mu}_{b}^{\prime} \in \mathcal{M}\left(c,\left(P^{d}, P_{-i}\right)\right)$. Also, writing $\hat{P}_{N}=\left(P^{d}, P_{-i}\right)$, we have $\mu_{b}^{\prime}(j)=b$ if and only if $t\left(\hat{P}_{j}\right) \in[c, b]$ and $\hat{\mu}_{b}^{\prime}(j)=b$ if and only if $t\left(\hat{P}_{j}\right) \in(c, b]$ for all $j \in N$. Therefore, by (28),

$$
\varphi_{c}\left(P^{d}, P_{-i}\right)=\beta_{\mu_{b}^{\prime}}([c, b])-\beta_{\hat{\mu}_{b}^{\prime}}((c, b]) .
$$

By (30), (31), (32), and the fact that $\hat{\mu}_{b}^{\prime}=\hat{\mu}_{b}$, we get

$$
\beta_{\mu_{b}}(\pi[c, b])<\beta_{\mu_{b}^{\prime}}(\pi[c, b]) .
$$

However, as (i) $\mu_{b}^{-1}(\hat{b})=\mu_{b}^{\prime-1}(\hat{b})$ for all $\hat{b} \in A_{L} \backslash\left\{b, b^{\prime}\right\}$ and (ii) $\mu_{b}^{\prime-1}(b) \subseteq \mu_{b}^{-1}(b)$, this contradicts condition (ii)(a) in Definition 4.2. 
Next we show the converse of Lemma 4.3.

Lemma 4.4. Let $\varphi$ be a unanimous and strategy-proof $R S C F$. Then there is a tree-monotonic collection of probability distributions $B=\left(\beta_{\mu}\right)_{\mu \in \mathcal{M}}$ such that $\varphi=\varphi^{B}$.

Proof. First note that $\varphi$ is uncompromising by Theorem 3.1. For every $\mu \in \mathcal{M}$ define $\beta_{\mu}=\varphi\left(P_{N}\right)$, where $P_{N} \in \mathcal{S}^{N}$ satisfies $t\left(P_{i}\right)=\mu(i)$ for all $i \in N$.

We first show that $B=\left(\beta_{\mu}\right)_{\mu \in \mathcal{M}}$ thus defined, is a tree-monotonic collection. Clearly, since $\varphi$ is unanimous, condition (i) in Definition 4.2 is satisfied. For condition (ii), let $\mu, \hat{\mu} \in \mathcal{M}$ and $i \in N$ such that $\mu(j)=\hat{\mu}(j)$ for all $j \in N \backslash\{i\}$ and let $P_{N}, \hat{P}_{N}$ such that $t\left(P_{k}\right)=\mu(k)$ and $t\left(\hat{P}_{k}\right)=\hat{\mu}(k)$ for all $k \in N$. Since $\varphi$ is uncompromising, $\varphi_{c}\left(P_{N}\right)=\varphi_{c}\left(\hat{P}_{N}\right)$ for all $c \notin\left[t\left(P_{i}\right), t\left(\hat{P}_{i}\right)\right]$, hence $\beta_{\mu}(c)=\beta_{\hat{\mu}}(c)$ for all $c \notin[\mu(i), \hat{\mu}(i)]$, i.e., condition (ii)(b) is satisfied. Moreover, by strategy-proofness of $\varphi$ we have for all $c \in\left[t\left(P_{i}\right), t\left(\hat{P}_{i}\right)\right]$ that $\varphi_{U\left(c, \hat{P}_{i}\right)}\left(\hat{P}_{N}\right) \geq \varphi_{U\left(c, \hat{P}_{i}\right)}\left(P_{N}\right)$. Since $\varphi_{z}\left(P_{N}\right)=\varphi_{z}\left(\hat{P}_{N}\right)$ for all $z \notin\left[t\left(P_{i}\right), t\left(\hat{P}_{i}\right)\right]$, this implies $\varphi_{\left[c, \hat{P}_{i}\right]}\left(\hat{P}_{N}\right) \geq \varphi_{\left[c, \hat{P}_{i}\right]}\left(P_{N}\right)$, and therefore $\beta_{\hat{\mu}}([c, \hat{\mu}(i)]) \geq \beta_{\mu}([c, \hat{\mu}(i)])$ for all $c \in[\mu(i), \hat{\mu}(i)]$. This proves condition (ii) (a).

Finally, we show that $\varphi=\varphi^{B}$. Let $P_{N} \in \mathcal{S}^{N}$ and $a \in A$. Let $\mu^{\prime}, \mu^{\prime \prime} \in \mathcal{M}\left(a, P_{N}\right)$ and $b \in A_{L}$ such that, for all $i \in N, \mu^{\prime}(i)=b$ if and only if $t\left(P_{i}\right) \in[a, b]$ and $\mu^{\prime \prime}(i)=b$ if and only if $t\left(P_{i}\right) \in(a, b]$. also, let $P_{N}^{\prime} \in \mathcal{S}^{N}$ such that $P_{i}^{\prime}=\mu^{\prime}(i)$ for all $i \in N$ and $P_{N}^{\prime \prime} \in \mathcal{S}^{N}$ such that $P_{i}^{\prime \prime}=\mu^{\prime \prime}(i)$ for all $i \in N$. Then

$$
\begin{aligned}
\varphi_{a}^{B}\left(P^{N}\right) & =\beta_{\mu^{\prime}}([a, b])-\beta_{\mu^{\prime \prime}}((a, b]) \\
& =\varphi_{[a, b]}\left(P_{N}^{\prime}\right)-\varphi_{(a, b]}\left(P_{N}^{\prime \prime}\right) \\
& =\varphi_{a}\left(P_{N}\right)
\end{aligned}
$$

where the last equality follows by uncompromisingness of $\varphi$. We conclude that $\varphi=\varphi^{B}$.

Lemmas 4.3 and 4.4 now imply the following result, which is the main result of the paper.

Theorem 4.1. Let $\mathcal{S}$ be a rich single-peaked domain. Then an $R S C F \varphi$ on $\mathcal{S}^{N}$ is unanimous and strategy-proof if and only if it is tree-monotonic.

\section{Final remarks}

The collection of tree-monotonic RSCFs contains deterministic functions, i.e., social choice functions that assign probability one to some alternative. It is not difficult to verify that these deterministic functions correspond to tree monotonic collections $B=\left(\beta_{\mu}\right)_{\mu \in \mathcal{M}}$ satisfying the following conditions: (1) for every $\mu \in \mathcal{M}, \beta_{\mu}(x)=1$ for some $x \in A$; (2) for every $\mu \in \mathcal{M}$, $\beta_{\mu}(b)=1$ if $\mu(i)=b$ for some $b \in A_{L}$ and all $i \in N$; and (3) for all $\mu, \hat{\mu} \in \mathcal{M}$ and $i \in N$ such that $\mu(j)=\hat{\mu}(j)$ for all $j \in N \backslash\{i\}$, we have: if $\beta_{\mu}(a)=1$ for some $a \in[\mu(i), \hat{\mu}(i)]$ then $\beta_{\hat{\mu}}(b)=1$ for some $b \in[a, \hat{\mu}(i)]$, and $\beta_{\mu}=\beta_{\hat{\mu}}$ otherwise.

We conclude with an example which shows that under the current domain assumptions not every tree-monotonic RSCF can be written as a convex combination of deterministic treemonotonic social choice functions. As mentioned earlier, on line-graphs these conditions are weaker than those in Peters et al. (2014). The question how these conditions should be strengthened to obtain all tree-monotonic RSCFs as convex combinations of deterministic tree-monotonic social choice functions is still open. 


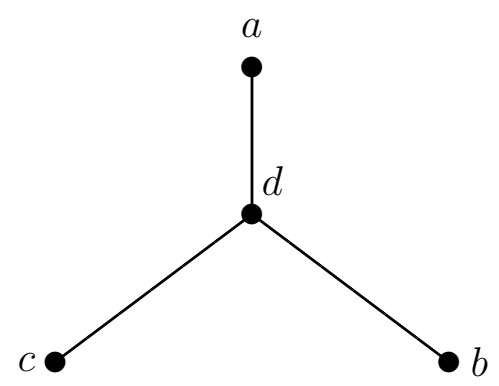

Figure 3: The tree in Example 5.1

Example 5.1. Let $N=\{1,2,3\}$ and $A=\{a, b, c, d\}$, with tree $E$ as in Figure 3 . We consider the (anonymous) tree-monotonic rule with tree-monotonic allocation functions as in the following table, in which $\beta_{(j, k, l)}$ denotes the probabilities assigned by the (anonymous) allocation function where $j$ agents are allocated to $a, k$ agents to $b$, and $l$ agents to $c$.

\begin{tabular}{|l|c|c|c|c|}
\hline & $a$ & $b$ & $c$ & $d$ \\
\hline$\beta_{(1,1,1)}$ & .5 & .3 & .2 & 0 \\
\hline$\beta_{(2,1,0)}$ & .7 & .3 & 0 & 0 \\
\hline$\beta_{(1,2,0)}$ & .5 & .4 & 0 & .1 \\
\hline$\beta_{(2,0,1)}$ & .7 & 0 & .2 & .1 \\
\hline$\beta_{(1,0,2)}$ & .5 & 0 & .3 & .2 \\
\hline$\beta_{(0,2,1)}$ & 0 & .4 & .2 & .4 \\
\hline$\beta_{(0,1,2)}$ & 0 & .3 & .3 & .4 \\
\hline
\end{tabular}

Additionally, $\beta_{(3,0,0)}, \beta_{(0,3,0)}$, and $\beta_{(0,0,3)}$ assign probability 1 to $a, b$, and $c$, respectively. The associated RSCF is denoted by $\psi$, and we will show that $\psi$ cannot be written as a convex combination of unanimous and strategy-proof deterministic rules.

Let $F$ be the set of all unanimous and strategy-proof deterministic rules on the singlepeaked domain on the tree given in Figure 3. Further, for an alternative $x$ and a profile $P_{N}$, let $F\left(x, P_{N}\right)$ be the set of all deterministic rules $f$ such that $f\left(P_{N}\right)=x$. By $\left(S_{1}, S_{2}, S_{3}\right)$, where $S_{1}, S_{2}, S_{3}$ are disjoint with union $N$, we mean a boundary profile where the top-alternatives of the agents in $S_{1}, S_{2}$, and $S_{3}$ are $a, b$, and $c$, respectively. Let $F_{1}=F(a,(\{1,2\},\{3\}, \emptyset))$, $F_{2}=F(b,(\{1,3\},\{2\}, \emptyset)), F_{3}=F(c,(\{1\},\{2\},\{3\})), F_{4}=F(b,(\{1,2\},\{3\}, \emptyset))$, and $F_{5}=$ $F(b,(\{1\},\{2,3\}, \emptyset))$. Then, by Theorem 4.1 , or more directly by uncompromisingness (Theorem 3.1), it follows that $F_{1} \cap F_{3}=\emptyset$ and $F_{2} \cap F_{3}=\emptyset$. Combining, we have

$$
\left(F_{1} \cup F_{2}\right) \cap F_{3}=\emptyset \text {. }
$$

Assume for contradiction that $\psi$ can be written as $\sum_{f \in F} \alpha_{f} f$. For $G \subseteq F$, let $\alpha_{G}=\sum_{f \in G} \alpha_{f}$. Then $\alpha_{F_{1} \cup F_{2}}=\alpha_{F_{1}}+\alpha_{F_{2}}-\alpha_{F_{1} \cap F_{2}}$ together with (34), yields $\alpha_{F_{1}}+\alpha_{F_{2}}-\alpha_{F_{1} \cap F_{2}}+\alpha_{F_{3}} \leq 1$. Since $\varphi=\sum_{f \in F} \alpha_{f} f$, it must be that $\alpha_{F_{1}}=\psi_{a}(\{1,2\},\{3\}, \emptyset), \alpha_{F_{2}}=\psi_{b}(\{1,3\},\{2\}, \emptyset)$, $\alpha_{F_{3}}=\psi_{c}(\{1\},\{2\},\{3\})$. Using the values given in the table we obtain

$$
\alpha_{F_{1} \cap F_{2}} \geq 0.2 \text {. }
$$

Since the rules in $F_{1}$ and $F_{4}$ give different outcomes ( $a$ and $b$, respectively) at the same profile $(\{1,2\},\{3\}, \emptyset)$, we have $F_{1} \cap F_{4}=\emptyset$. Moreover, by uncompromisingness, $F_{2} \subseteq F_{5}$ and $F_{4} \subseteq F_{5}$, and hence $F_{2} \cup F_{4} \subseteq F_{5}$. Because $F_{1} \cap F_{4}=\emptyset$, we have

$$
\left(F_{1} \cap F_{2}\right) \cap F_{4}=\emptyset \text {. }
$$


Also, because $F_{2} \cup F_{4} \subseteq F_{5}$,

$$
\left(F_{1} \cap F_{2}\right) \cup F_{4} \subseteq F_{5} .
$$

Combining (36) and (37), we have $\alpha_{F_{1} \cap F_{2}}+\alpha_{F_{4}} \leq \alpha_{F_{5}}$. By (35) and the table, $\alpha_{F_{1} \cap F_{2}}+\alpha_{F_{4}} \geq$ 0.5 , and hence $\alpha_{F_{5}} \geq 0.5$. However, this contradicts the fact that $\alpha_{F_{5}}=0.4$. This proves that $\psi$ cannot be written as a convex combination of deterministic rules.

\section{References}

Barberà S, Gul F, Stacchetti E (1993) Generalized median voter schemes and committees. Journal of Economic Theory 61:262-289

Black D (1948) On the rationale of group decision-making. Journal of Political Economy $56: 23-34$

Border K, Jordan J (1983) Straightforward elections, unanimity and phantom voters. Review of Economic Studies 50:153-170

Chatterji S, Massó J (2015) On strategy-proofness and the salience of single-peakedness. Working paper

Chatterji S, Zeng H (2015) On random social choice function with the tops-only property. Working paper

Chatterji S, Sen A, Zeng H (2016) A characterization of single-peaked preferences via random social choice functions. Theoretical Economics 11:711-733

Ehlers L, Peters H, Storcken A (2002) Strategy-proof probabilistic decision schemes for one-dimensional single-peaked preferences. Journal of Economic Theory 105:408-434

Gibbard A (1977) Manipulation of voting schemes that mix voting with chance. Econometrica 45:665-681

Inada KI (1964) A note on the simple majority rule. Econometrica 32:525-531

Moulin H (1980) On strategy-proofness and single peakedness. Public Choice 35:437-455

Peters H, Roy S, Sen A, Storcken T (2014) Probabilistic strategy-proof rules over singlepeaked domains. Journal of Mathematical Economics 52:123-127

Sen, A (2011) The Gibbard random dictatorship theorem: A generalization and a new proof. Spanish Economic Review, SERIEs 2(4):515-527 